\title{
HAUSDORFF DIMENSION, PROJECTIONS, AND THE FOURIER TRANSFORM
}

\author{
Pertti Mattila
}

Abstract

This is a survey on transformation of fractal type sets and measures under orthogonal projections and more general mappings.

\section{Contents}

Foreword 3

Acknowledgements 4

1. Hausdorff dimension and orthogonal projections 4

2. Cantor sets, tubular neighborhoods, and isoperimetric problems

3. Fourier transforms of measures with finite energy 16

4. Besicovitch sets 24

5. Generalized projections of Peres and Schlag 29

6. Visibility problems 36

$\begin{array}{ll}\text { 7. Open problems } & 39\end{array}$

References $\quad 42$

\section{Foreword}

This is a survey on results and methods on the behaviour of Hausdorff dimension under projection-type mappings and related topics. A particular emphasis will be in the role of the Fourier transform in such problems. It is not meant to be exhaustive. We concentrate on only a few topics and try to explain some of the main ideas of the proofs in these cases.

The first pivotal result on orthogonal projections and Hausdorff measures was Besicovitch's theorem from 1938. It says that if $E \subset \mathbb{R}^{2}$ is measurable with respect to the 1-dimensional Hausdorff measure $\mathcal{H}^{1}, 0<$ $\mathcal{H}^{1}(E)<\infty$, and $E$ is purely unrectifiable in the sense that $\mathcal{H}^{1}(E \cap \Gamma)=0$

2000 Mathematics Subject Classification. 28A75; Secondary: 28A78, 42B10.

Key words. Hausdorff measure and dimension, projection, Fourier transform. 
for every rectifiable curve $\Gamma$, then the orthogonal projection of $E$ on almost every line through the origin has zero length. We shall give a proof of this in Section 2 for some special Cantor sets due to Peres and Solomyak in [PSo] but otherwise we shall leave integral dimensional sets aside and concentrate on sets whose Hausdorff dimension could be anything. In this context the fundamental theorem is due to Marstrand in $[\mathbf{M}]$ from 1954. We shall present it and its proof in Section 1. In Section 2 we develop these methods further to estimate measures of the orthogonal projections of tubular neighborhoods and the averages of measures of projections. There we shall also discuss projection properties of some self-similar Cantor sets. Fourier transform enters in Section 3 and we first illustrate its power in the proof of Falconer's distance set theorem and its generalization in $\mathbb{R}^{2}$ by Wolff. In Section 4 we investigate Besicovitch sets. They are sets of Lebesgue measure zero which contain a line in every direction. In Section 5 we discuss the powerful and elegant theory of Peres and Schlag to treat projection-type mappings in a general setting. In addition to its application to orthogonal projections, we briefly discuss pinned distance sets and Bernoulli convolutions. In Section 6 we study various concepts of visibility. Finally, in Section 7 we collect several open problems.

Acknowledgements. This survey is based on a mini-course I gave in Universitat Autònoma de Barcelona in January-February, 2003. I would like to thank the inspiring audience for their many comments and questions. In particular, I would like to thank Joan Orobitg for practical arrangements and Joan Verdera for inviting me to submit this paper to Publicacions Matemàtiques. I am also indebted to Departament de Matemàtiques de Universitat Autònoma and CRM for financial support and wonderful working environment. I wish to extend my thanks also to Francesco Serra Cassano and Dipartimento di Matematica, Università di Trento, where this paper was completed. Finally, I would like to thank Maarit Järvenpää, Toby O'Neil, Yuval Peres and Ville Suomala for many useful comments on the first version of this paper.

\section{Hausdorff dimension and orthogonal projections}

Notation. By $\mu$ and $\nu$ we shall always mean locally finite Borel measures in $\mathbb{R}^{n}$, or in a metric space, that is, outer measures such that balls have finite measure and Borel sets are measurable. The support of a measure $\mu$ is the closed set

$$
\operatorname{spt} \mu=\{x \in \mathbb{R}: \mu(B(x, r))>0 \text { for all } r>0\},
$$


where $B(x, r)$ is the closed ball with centre $x$ and radius $r$. We set for $A \subset \mathbb{R}^{n}$,

$$
\begin{aligned}
\mathcal{M}(A) & =\{\mu: \operatorname{spt} \mu \subset A, \text { spt } \mu \text { is compact, } 0<\mu(A)<\infty\}, \\
\mathcal{M}_{1}(A) & =\{\mu \in \mathcal{M}(A): \mu(A)=1\} .
\end{aligned}
$$

$\mathcal{L}^{n}$ is the Lebesgue measure in $\mathbb{R}^{n}$, and also in an $n$-plane in $\mathbb{R}^{p}$. For $0 \leq s \leq n$ we denote by $\mathcal{H}^{s}$ the $s$-dimensional Hausdorff measure defined by

$$
\mathcal{H}^{s}(A)=\lim _{\delta \rightarrow 0} \inf \left\{\sum_{i=1}^{\infty} \operatorname{diam}\left(E_{i}\right)^{s}: A \subset \bigcup_{i=1}^{\infty} E_{i}, \operatorname{diam}\left(E_{i}\right)<\delta\right\} .
$$

Then, as one easily checks,

$$
\mathcal{H}^{s}(A)<\infty, \quad \text { and } s<t, \quad \Longrightarrow \mathcal{H}^{t}(A)=0 .
$$

So we can define the Hausdorff dimension of $A$ as

$$
\operatorname{dim} A=\sup \left\{s: \mathcal{H}^{s}(A)=\infty\right\}=\inf \left\{t: \mathcal{H}^{t}(A)=0\right\}
$$

A classical result from the 1930's called Frostman's lemma (cf. [M6, Theorem 8.8]), says that for a Borel set $A \subset \mathbb{R}^{n}, \mathcal{H}^{s}(A)>0$ if and only if there is $\mu \in \mathcal{M}(A)$ such that

$$
\mu(B(x, r)) \leq r^{s} \text { for } x \in \mathbb{R}^{n} \text { and } r>0 .
$$

That the existence of such a measure implies $\mathcal{H}^{s}(A)>0$ is very easy. The converse is more difficult. In particular, if $A$ is a general Borel set, and not for example closed, one needs the theory of analytic (Suslin) sets.

From (1.1) we can get an integral condition for the Hausdorff dimension. Namely, using the well-known formula $\int f d \mu=\int_{0}^{\infty} \mu(\{x: f(x)>$ $t\}) d t$ for non-negative $\mu$ measurable functions $f$, we have

$$
s \int_{0}^{\infty} \frac{\mu(B(x, r))}{r^{s+1}} d r=\int|x-y|^{-s} d \mu y
$$

Integrating also with respect to $x$ we get the $s$-energy of $\mu$ :

$$
I_{s}(\mu)=\iint|x-y|^{-s} d \mu y d \mu x
$$

Using these relations, Frostman's lemma leads to

$$
\operatorname{dim} A=\sup \left\{s: \exists \mu \in \mathcal{M}(A) \text { such that } I_{s}(\mu)<\infty\right\}
$$

for Borel sets $A \subset \mathbb{R}^{n}$. Defining the Riesz $s$-capacity of $A$ by

$$
C_{s}(A)=\sup \left\{I_{s}(\mu)^{-1}: \mu \in \mathcal{M}_{1}(A)\right\}
$$


we can rewrite this as

$$
\operatorname{dim} A=\sup \left\{s: C_{s}(A)>0\right\} .
$$

Our main interest in this section will be in the Hausdorff dimensions of orthogonal projections of Borel sets. We denote

$$
G(n, m)=\left\{V: V \text { is an } m \text {-dimensional linear subspace of } \mathbb{R}^{n}\right\},
$$

and by

$$
P_{V}: \mathbb{R}^{n} \longrightarrow V \text {, the orthogonal projection onto } V \text {. }
$$

To make it easier to follow and more concrete, we shall usually give proofs only for lines in the plane and projections onto them. To $\theta \in[0, \pi)$ we associate the unit vector

$$
\boldsymbol{\theta}=(\cos \theta, \sin \theta)
$$

the line

$$
L_{\theta}=\{t \boldsymbol{\theta}: t \in \mathbb{R}\},
$$

and the orthogonal projection "onto $L_{\theta}$ "

$$
p_{\theta}: \mathbb{R}^{2} \longrightarrow \mathbb{R}, \quad p_{\theta} x=\boldsymbol{\theta} \cdot x .
$$

By $x \mapsto(\boldsymbol{\theta} \cdot x) \boldsymbol{\theta}$ we would get the real projection onto $L_{\theta}$, but the above notation is simpler and, of course is essentially the same thing.

The theory of Haar measure gives a natural measure on $G(n, m)$. Letting $\theta_{n}$ be the Haar measure on the orthogonal group $O(n)$ of $\mathbb{R}^{n}$ we can define

$$
\gamma_{n, m}(A)=\theta_{n}\left(\left\{g: g\left(V_{0}\right) \in A\right\}\right), \quad A \subset G(n, m),
$$

where $V_{0}$ is some fixed $m$-plane in $G(n, m)$.

In the plane $\gamma_{2,1}$ can be given quite easily: we identify it with the normalized Lebesgue measure $\frac{1}{\pi} \mathcal{L}^{1} \mid[0, \pi)$ on $[0, \pi)$, or instead with the normalized length measure on the half circle $\{\boldsymbol{\theta}: \theta \in[0, \pi)\}$. Similarly in $\mathbb{R}^{n}$ the normalized area measure (or $\mathcal{H}^{n-1}$ ) on $S^{n-1}$ gives $\gamma_{n, 1}$, and also $\gamma_{n, n-1}$ via orthogonal complements.

Our basic problem is: what can we say about the relations between the Hausdorff measures and dimensions of the projections $P_{V}(A), V \in$ $G(n, m)$, and those of $A$ ? One direction is clear. As $P_{V}$ does not increase distances; $\left|P_{V} x-P_{V} y\right| \leq|x-y|$, we always have

$$
\mathcal{H}^{s}\left(P_{V}(A)\right) \leq \mathcal{H}^{s}(A) \text { and } \operatorname{dim} P_{V}(A) \leq \operatorname{dim} A .
$$

To understand some of the difficulties in this problem, let us look at some examples. 
Cantor sets $\boldsymbol{C}_{\boldsymbol{d}}$. For $0<d<\frac{1}{2}$, let $C_{d}$ be the "four corner Cantor set"

$$
C_{d}=\bigcap_{k=1}^{\infty} U_{k}^{d}, \quad U_{k}^{d}=\bigcup_{i=1}^{4^{k}} Q_{k, i}
$$

Here each $Q_{k, i}$ is a closed square of side-length $d^{k}$, and they are defined as follows. First the $Q_{1, i}$ s are the four squares in the four corners of the unit square $[0,1] \times[0,1] ;[0, d] \times[0, d],[0, d] \times[1-d, 1],[1-d, 1] \times[0, d]$ and $[1-d, 1] \times[1-d, 1]$. If the squares $Q_{k, i}, i=1, \ldots, 4^{k}$, have been constructed, the $Q_{k+1, j}$ s are obtained in the same way inside and in the corners of the $Q_{k, i} \mathrm{~s}$.

If we want to determine the Hausdorff dimension of $C_{d}$, a natural guess is that essentially best coverings are those provided by the squares $Q_{k, i}$. For them we have

$$
\sum_{i=1}^{4^{k}} \operatorname{diam}\left(Q_{k, i}\right)^{s}=4^{k} 2^{\frac{s}{2}} d^{k s}=2^{\frac{s}{2}}\left(4 d^{s}\right)^{k} .
$$

Defining $s_{d}$ by

$$
4 d^{s_{d}}=1 \text {, i.e., } s_{d}=\frac{\log 4}{\log \left(\frac{1}{d}\right)},
$$

we see that this sum tends to zero as $k \rightarrow \infty$, if $s>s_{d}$, it is $2^{\frac{s}{2}}$ for all $k$, if $s=s_{d}$, and it tends to $\infty$ if $s<s_{d}$. The first of these facts implies that $\mathcal{H}^{s}\left(C_{d}\right)=0$ if $s>s_{d}$, whence $\operatorname{dim} C_{d} \leq s_{d}$, the second that $\mathcal{H}^{s_{d}}\left(C_{d}\right) \leq 2^{\frac{s}{2}}$. None of these really implies directly that $\mathcal{H}^{s_{d}}\left(C_{d}\right)>0$, nor that $\operatorname{dim} C_{d} \geq s_{d}$, because for this we should use arbitrary coverings. But arbitrary coverings are not too hard to reduce to these special ones and we have

$$
0<\mathcal{H}^{s_{d}}\left(C_{d}\right)<\infty \text { and } \operatorname{dim} C_{d}=s_{d}
$$

When we look at the projections $p_{\theta}\left(C_{d}\right)$, we notice immediately that when $\theta=0$ or $\theta=\frac{\pi}{2}$, that is, when we project into the coordinate axis, we get Cantor sets whose dimensions are $\frac{\log 2}{\log \left(\frac{1}{d}\right)}=\frac{1}{2} s_{d}$. Looking more carefully at these projections with different angles $\theta$ we find easily a countable dense set of angles $\theta$ for which $p_{\theta}\left(C_{d}\right)$ is a Cantor set in $\mathbb{R}$ with dimension strictly less than $s_{d}$. This happens always when $p_{\theta}$ maps two different squares $Q_{k, i}$ exactly onto the same interval. However, we shall see now that this behaviour is exceptional: typically $\operatorname{dim}_{p_{\theta}}\left(C_{d}\right)=s_{d}$, if $s_{d} \leq 1$, and $\mathcal{L}^{1}\left(p_{\theta}\left(C_{d}\right)\right)>0$, if $s_{d}>1$. The following theorem is due to Marstrand from $[\mathbf{M}]$. Potential theoretic methods for the proof were first used by Kaufman in [K1]. 
Theorem 1.1. Let $A \subset \mathbb{R}^{n}$ be a Borel set with $\operatorname{dim} A=s$.

(1) If $s \leq m, \operatorname{dim} P_{V}(A)=s$ for $\gamma_{n, m}$ almost all $V \in G(n, m)$.

(2) If $s>m, \mathcal{L}^{m}\left(P_{V}(A)\right)>0$ for $\gamma_{n, m}$ almost all $V \in G(n, m)$.

Proof (for $m=1, n=2$ ): (1) We can assume $s>0$. Let $0<t<s$. By (1.2), we can find $\mu \in \mathcal{M}(A)$ such that $I_{t}(\mu)<\infty$. Let $p_{\theta} \mu$ be the image of $\mu$ under $p_{\theta}$; that is,

$$
p_{\theta} \mu(B)=\mu\left(p_{\theta}^{-1}(B)\right) \text { for } B \subset \mathbb{R} .
$$

Then $p_{\theta} \mu \in \mathcal{M}\left(p_{\theta} A\right)$ and

$$
\begin{aligned}
\int_{0}^{\pi} I_{t}\left(p_{\theta} \mu\right) d \theta & =\int_{0}^{\pi} \iint|u-v|^{-t} d\left(p_{\theta} \mu(u)\right) d\left(p_{\theta} \mu(v)\right) d \theta \\
& =\int_{0}^{\pi} \iint\left|p_{\theta} x-p_{\theta} y\right|^{-t} d \mu x d \mu y d \theta \\
& =\iiint_{0}^{\pi}|\boldsymbol{\theta} \cdot(x-y)|^{-t} d \theta d \mu x d \mu y=c(t) I_{t}(\mu) .
\end{aligned}
$$

Here the second equation follows easily from the definition of the image measure $p_{\theta} \mu$, the third from Fubini's theorem, and the fourth from an elementary computation which gives for $z \in \mathbb{R}^{2}$,

$$
\int_{0}^{\pi}|\boldsymbol{\theta} \cdot z|^{-t} d \theta=\int_{0}^{\pi}(\cos \theta)^{-t}|z|^{-t} d \theta=c(t)|z|^{-t} .
$$

Here $c(t)<\infty$ since $t<1$. Thus

$$
I_{t}\left(p_{\theta} \mu\right)<\infty \text { for almost all } \theta,
$$

whence by (1.2), $\operatorname{dim} p_{\theta}(A) \geq t$ for almost $\theta$. Since $t<s$ was arbitrary, we have $\operatorname{dim} p_{\theta}(A) \geq s$ for almost all $\theta$. Finally the opposite inequality $\operatorname{dim} p_{\theta}(A) \leq s=\operatorname{dim} A$ always holds, recall (1.4), and we have proved (1).

To prove (2) we use the following proposition, see e.g. [M6, Theorem 2.12].

Proposition. Let $\mu$ be a locally finite Borel measure in $\mathbb{R}^{n}$. Then $\mu$ is absolutely continuous with respect to the Lebesgue measure, $\mu \ll \mathcal{L}^{n}$, if and only if

$$
\liminf _{r \rightarrow 0} r^{-n} \mu(B(x, r))<\infty \text { for } \mu \text { almost all } x \in \mathbb{R}^{n} .
$$


Note that if we replaced " $\mu$ almost all" by " $\mathcal{L}^{n}$ almost all", then (1.6) would be true for any $\mu$. This proposition is a consequence of Vitali's covering theorem for $\mu$ which in turn is based on Besicovitch's covering theorem.

To prove (2) we first pick $\mu \in \mathcal{M}(A)$ with $I_{1}(\mu)<\infty$. Then by Fatou's lemma, the definition of $p_{\theta} \mu$, and Fubini's theorem,

$$
\begin{aligned}
& \int_{0}^{\pi} \int \liminf _{r \rightarrow 0} \frac{1}{2 r}\left(p_{\theta} \mu\right)([u-r, u+r]) d\left(p_{\theta} \mu(u)\right) d \theta \\
& \quad \leq \liminf _{r \rightarrow 0} \frac{1}{2 r} \int_{0}^{\pi} \int \mu(\{y:|\boldsymbol{\theta} \cdot(x-y)| \leq r\}) d \mu x d \theta \\
& \quad=\liminf _{r \rightarrow 0} \frac{1}{2 r} \iint \mathcal{L}^{1}(\{\theta \in[0, \pi):|\boldsymbol{\theta} \cdot(x-y)| \leq r\}) d \mu y d \mu x \\
& \quad=I_{1}(\mu)<\infty .
\end{aligned}
$$

The last equation comes from the elementary fact that

$$
\mathcal{L}^{1}(\{\theta \in[0, \pi):|\boldsymbol{\theta} \cdot z| \leq r\}) \sim \frac{2 r}{|z|}
$$

for small $r$. Using this and the above proposition we get $p_{\theta} \mu \ll \mathcal{L}^{1}$ for almost all $\theta$. Since spt $p_{\theta} \mu \subset p_{\theta}(A)$, this yields $\mathcal{L}^{1}\left(p_{\theta} A\right)>0$ for almost all $\theta$ and proves the theorem.

The proof of (2) actually gives more. We have shown there that if $I_{1}(\mu)<\infty$, then for almost all $\theta, p_{\theta} \mu \ll \mathcal{L}^{1}$ with Radon-Nikodým derivative in $L^{2}$. The Radon-Nikodým derivative is

$$
D\left(p_{\theta} \mu\right)(u)=\lim _{r \rightarrow 0} \frac{p_{\theta} \mu([u-r, u+r])}{2 r}
$$

for $\mathcal{L}^{1}$ almost all $u$. We also used here the fact

$$
\int D\left(p_{\theta} \mu\right) d\left(p_{\theta} \mu\right)=\int D\left(p_{\theta} \mu\right)^{2} d \mathcal{L}^{1}
$$

We state the inequality we derived using this notation:

$$
\int_{0}^{\pi} \int_{\mathbb{R}} D\left(p_{\theta} \mu\right)^{2} d \mathcal{L}^{1} d \theta \leq I_{1}(\mu) .
$$

As usual, we shall often identify an absolutely continuous measure with its Radon-Nikodým derivative. Thus, for example, writing $p_{\theta} \mu \in L^{2}$ we mean that $p_{\theta} \mu \ll \mathcal{L}^{1}$ and $D\left(p_{\theta} \mu\right) \in L^{2}$. 
Another way to prove (2) is to use Fourier transforms and Plancherel's theorem. A rather straightforward computation (see [F2, p. 82]) shows that

$$
\int_{0}^{\pi} \int_{\mathbb{R}}\left|\left(p_{\theta} \mu\right)^{\wedge}(u)\right|^{2} d u d \theta \leq C I_{1}(\mu),
$$

which again gives that $p_{\theta} \mu \in L^{2}$ for almost all $\theta$.

The proof of Theorem 1.1(1) gives also the following sharpening in terms of capacities: if $0<s<m$ and $C_{s}(A)>0$, then $C_{s}\left(P_{V}(A)\right)>0$ for $\gamma_{n, m}$ almost all $V \in G(n, m)$. In (2) the assumption $\operatorname{dim} A>m$ can be replaced by $C_{m}(A)>0$. Dijkstra and van Mill in $[\mathbf{D M}]$ and Monterie in [Mo] studied also capacities of projections.

One can say something more about the exceptional sets in Theorem 1.1:

Theorem 1.2. Let $A \subset \mathbb{R}^{2}$ be a Borel set with $\operatorname{dim} A=s$.

(1) If $s \leq 1, \operatorname{dim}\left\{\theta: \operatorname{dim} p_{\theta}(A)<s\right\} \leq s$.

(2) If $s>1, \operatorname{dim}\left\{\theta: \mathcal{L}^{1}\left(p_{\theta}(A)\right)=0\right\} \leq 2-s$.

The proof of (1) is similar to that of Theorem 1.1(1) using Frostman's lemma on the exceptional set of directions. This is due to Kaufman [K1]. The second part (2) is due to Falconer [F1]. The proof of (2) uses the Fourier transform and no proof without it is known. We return to this later.

Kaufman and I have shown in $[\mathbf{K M}]$ by some number theoretic examples that $s$ in (1) is sharp. Similar examples can also be used to show that $2-s$ is sharp in (2).

Let us return to the Cantor sets $C_{d}$. We know now that $\operatorname{dim} p_{\theta}\left(C_{d}\right)=$ $s_{d}$ for almost all $\theta$ if $d \leq \frac{1}{4}$. But what about the measures $\mathcal{H}^{s_{d}}\left(C_{d}\right)$ ? When $d=\frac{1}{4}$ (and $\left.s_{d}=1\right), C_{\frac{1}{4}}$ is an example of a purely unrectifiable set (i.e., $\mathcal{H}^{1}\left(C_{\frac{1}{4}} \cap \Gamma\right)=0$ for every rectifiable curve $\Gamma$ ) with positive and finite $\mathcal{H}^{1}$ measure. By a general theorem of Besicovitch (see [F2, Theorem 6.13] or [M6, Theorem 18.1]) we have then that $\mathcal{H}^{1}\left(p_{\theta}\left(C_{\frac{1}{4}}\right)\right)=$ 0 for almost all $\theta$. We give an elementary proof of this (for $C_{\frac{1}{4}}$ ) in the next section. Peres, Simon and Solomyak proved in [PSS1] that this remains true for $\frac{1}{6}<d<\frac{1}{4}$, that is, then $\mathcal{H}^{s_{d}}\left(p_{\theta}\left(C_{d}\right)\right)=0$ for almost all $\theta$.

If $d<\frac{1}{9}$, it is not too hard to show that $\mathcal{H}^{s_{d}}\left(p_{\theta}\left(C_{d}\right)\right)>0$ for almost all $\theta$. We give a few hints for this. Look at

$$
C_{d}-C_{d}=\left\{x-y: x, y \in C_{d}\right\} .
$$


When $d \geq \frac{1}{3}$, it is a square. When $d<\frac{1}{3}$, it is a self-similar Cantor set of dimension $\frac{\log 9}{\log \left(\frac{1}{d}\right)}$. Hence if $d<\frac{1}{9}, \mathcal{H}^{1}\left(C_{d}-C_{d}\right)=0$, so also

$$
D=\left\{\frac{x-y}{|x-y|}: x, y \in C_{d}, x \neq y\right\} \subset S^{1}
$$

has zero 1-dimensional measure. This is the set of directions between the points of $C_{d}$. Using the self-similarity of $C_{d}$ one can show that $D$ is closed. This implies that if $\boldsymbol{\theta} \in S^{1} \backslash D, p_{\theta} \mid C_{d}$ is injective with Lipschitz inverse, which gives $\mathcal{H}^{s_{d}}\left(p_{\theta}\left(C_{d}\right)\right)>0$. It is not known if the same is true for $\frac{1}{9} \leq d \leq \frac{1}{6}$.

\section{Cantor sets, tubular neighborhoods, and isoperimetric problems}

We return to the one-dimensional Cantor set $C_{\frac{1}{4}}$ and give a proof that it projects to zero length in almost all directions. This proof is due to Peres, Simon and Solomyak [PSS1], and it was made quantitative by Peres and Solomyak in [PSo] (a very nice exposition is given in [PSS2]). Another proof is given by Kenyon in $[\mathbf{K e}]$. He gives also a sharper result, which in particular implies that there are only countably many directions $\theta$ for which $\mathcal{L}^{1}\left(p_{\theta}\left(C_{d}\right)\right)>0$. By simple geometric inspections one checks that the set of such directions is countably infinite and dense.

Denote now $C=C_{\frac{1}{4}}$. We can write

$$
C=\bigcup_{i=1}^{4}\left(\frac{1}{4} C+c_{i}\right)
$$

where $c_{1}=(0,0), c_{2}=\left(\frac{3}{4}, 0\right), c_{3}=\left(0, \frac{3}{4}\right), c_{4}=\left(\frac{3}{4}, \frac{3}{4}\right)$. Hence

$$
p_{\theta}(C)=\bigcup_{i=1}^{4}\left(\frac{1}{4} p_{\theta}(C)+\boldsymbol{\theta} \cdot c_{i}\right) \subset \mathbb{R} .
$$

Let us first look more generally at this type of self-similar subsets of $\mathbb{R}$. Let $K \subset \mathbb{R}$ be compact such that for some integer $m \geq 2$ and some $d_{1}, \ldots, d_{m} \in \mathbb{R}\left(d_{i} \neq d_{j}\right.$ for $\left.i \neq j\right)$

$$
K=\bigcup_{i=1}^{m} K_{i} \text { with } K_{i}=\frac{1}{m} K+d_{i} .
$$

Lemma 1. (1) $\mathcal{L}^{1}\left(K_{i} \cap K_{j}\right)=0$ for $i \neq j$.

(2) $K_{i} \cap K_{j} \neq \emptyset$ for some $i \neq j$. 
(1) follows easily from $\mathcal{L}^{1}\left(K_{i}\right)=\frac{1}{m} \mathcal{L}^{1}(K)$.

We leave the proof of (2) as an exercise (or see [PSS2]). One starting point could be to assume that $K_{i} \cap K_{j}=\emptyset$ for $i \neq j$, to conclude that for some $\varepsilon>0$ also the $\varepsilon$-neighborhoods of the $K_{i}$ 's are disjoint and continue from this using the scaling properties.

Since

$$
K_{i}=\frac{1}{m} K+d_{i}=\frac{1}{m}\left(\bigcup_{j=1}^{m}\left(\frac{1}{m} K+d_{j}\right)\right)+d_{i}=\bigcup_{j=1}^{m} K_{i, j},
$$

where $K_{i j}=\frac{1}{m^{2}} K+\frac{1}{m} d_{j}+d_{i}$, we can write $K$ also as the union of the $m^{2}$ sets $K_{i j}$. Set

$$
\begin{aligned}
I & =\{1, \ldots, m\}, \\
I^{k} & =\left\{u: u=\left(i_{1}, \ldots, i_{k}\right), i_{j} \in I\right\}, \quad k=1,2, \ldots .
\end{aligned}
$$

Then for each $k$,

$$
K=\bigcup_{u \in I^{k}} K_{u}, \text { where } K_{u}=m^{-k} K+d_{u} .
$$

The sets $K_{u}$ were defined above for $k=1,2$, and the general case should be clear from this.

The following notion is due to Bandt and Graf, [BG].

Definition. Let $\varepsilon>0$. We say that $K_{u}$ and $K_{v}$ are $\varepsilon$-relatively close if $u, v \in I^{k}$ for some $k, u \neq v$, and

$$
K_{v}=K_{u}+x
$$

for some $x \in \mathbb{R}$ with $|x| \leq \varepsilon \operatorname{diam}\left(K_{u}\right)$.

Note that for $u, v \in I^{k}, K_{v}$ is always a translation of $K_{u}$, but we are now requiring that we don't have to translate too much.

Lemma 2. If for every $\varepsilon>0$ there are $k$ and $u, v \in I^{k}$ with $u \neq v$ such that $K_{u}$ and $K_{v}$ are $\varepsilon$-relatively close, then $\mathcal{L}^{1}(K)=0$.

To prove this suppose $\mathcal{L}^{1}(K)>0$. Then there is some interval $I$ such that $\mathcal{L}^{1}(K \cap I)>0.9 \mathcal{L}^{1}(I)$. Pick small $\varepsilon>0$ and $K_{u}$ and $K_{v}, u, v \in I^{k}$, $u \neq v$, which are $\varepsilon$-relatively close. Then the intervals $m^{-k} I+d_{u}$ and $m^{-k} I+d_{v}$ overlap a lot (since $\varepsilon$ is small). But $K_{u}$ and $K_{v}$ fill $90 \%$ of each of them, which is impossible since $\mathcal{L}^{1}\left(K_{u} \cap K_{v}\right)=0$ (by an iteration of Lemma 1(2)). We leave the details to the reader. 
We return now to the proof that $\mathcal{L}^{1}\left(p_{\theta}(C)\right)=0$ for almost all $\theta$. Set $p_{\theta}(C)=C^{\theta}$ to make it more convenient to use the notations $C_{u}^{\theta}$ from above. For $\varepsilon>0$ let

$$
\begin{aligned}
& V_{\varepsilon}=\{\theta \in[0, \pi): \exists k, u, v \text { such that } u, v \in I^{k}, u \neq v \\
&\text { and } \left.C_{u}^{\theta} \text { and } C_{v}^{\theta} \text { are } \varepsilon \text {-relatively close }\right\} .
\end{aligned}
$$

It follows from Lemma 2 that it suffices to show that for every $\varepsilon>0$,

$$
\mathcal{L}^{1}\left([0, \pi) \backslash V_{\varepsilon}\right)=0 .
$$

(Then also $\mathcal{L}^{1}\left(S^{1} \backslash \bigcap_{\varepsilon>0} V_{\varepsilon}\right)=\mathcal{L}^{1}\left(S^{1} \backslash \bigcap_{j=1}^{\infty} V_{\frac{1}{j}}\right)=0$.) So let $\varepsilon>0$ and $\theta \in[0, \pi)$. By Lemma $1(2), C_{i}^{\theta} \cap C_{j}^{\theta} \neq \emptyset$ for some $i \neq j$. This means that there are $x \in C_{i}$ and $y \in C_{j}$ such that $p_{\theta} x=p_{\theta} y$. Let $k>1$ be an integer. Then $x \in C_{u}$ and $y \in C_{v}$ for some $u, v \in I^{k}$ with $u \neq v$. Let $\theta_{o} \in[0, \pi)$ be such that $p_{\theta_{0}}\left(C_{u}\right)=p_{\theta_{0}}\left(C_{v}\right)$ (that is, $p_{\theta_{0}}$ maps the squares of side-length $4^{-k}$ which contain $C_{u}$ and $C_{v}$ onto the same interval). Then $C_{u}^{\theta_{0}}$ and $C_{v}^{\theta_{0}}$ are "0-relatively close", and a simple geometric inspection shows that $C_{u}^{\varphi}$ and $C_{v}^{\varphi}$ are $\varepsilon$-relatively close when $\left|\varphi-\theta_{0}\right|<b \varepsilon 4^{-k}$, where $b$ is a constant. Hence, with another constant $c$, $\left[\theta-c 4^{-k}, \theta+c 4^{-k}\right] \cap V_{\varepsilon}$ contains an interval of length $b \varepsilon 4^{-k}$. Since this is true for every $k$, it follows that $\mathcal{L}^{1}\left([0, \pi) \backslash V_{\varepsilon}\right)=0$ as required.

Using the notion of $\varepsilon$-relative closeness more effectively Peres and Solomyak have given the following quantitative version of the above result in [PSo]. For $y \geq 1$ define

$$
\log _{*} y=\min \{n \in \mathbb{N}: \underbrace{\log \log \ldots \log y}_{n} \leq 1\} .
$$

Then $\log _{*} y \rightarrow \infty$ as $y \rightarrow \infty$ but extremely slowly. They proved that with some positive constants $C$ and $a$,

$$
\int_{0}^{\pi} \mathcal{L}^{1}\left(p_{\theta}\left(U_{k}^{\frac{1}{4}}\right)\right) d \theta \leq C \exp \left(-a \log _{*} k\right)
$$

where $U_{k}^{\frac{1}{4}}$ is the union of the $4^{k}$ squares as in (1.5).

We now use (1.7) to derive a lower bound. Let $\mu \in \mathcal{M}_{1}\left(\mathbb{R}^{2}\right)$ and denote $F=\operatorname{spt} \mu$. If $p_{\theta} \mu \ll \mathcal{L}^{1}$, we have by Schwartz's inequality

$$
1=p_{\theta}(F)=\left(\int_{p_{\theta}(F)} D\left(p_{\theta} \mu\right) d \mathcal{L}^{1}\right)^{2} \leq \mathcal{L}^{1}\left(p_{\theta}(F)\right) \int D\left(p_{\theta} \mu\right)^{2} d \mathcal{L}^{1},
$$


and

$$
\begin{aligned}
\int_{0}^{\pi} \mathcal{L}^{1}\left(p_{\theta}(F)\right)^{-1} d \theta & \leq \int_{0}^{\pi} \int D\left(p_{\theta} \mu\right)^{2} d \mathcal{L}^{1} d \theta \\
& \leq I_{1}(\mu)
\end{aligned}
$$

by (1.7). This gives

$$
\int_{0}^{\pi} \mathcal{L}^{1}\left(p_{\theta}(F)\right) d \theta \geq \frac{\pi^{2}}{I_{1}(\mu)}
$$

(This is just Schwartz's inequality: $f \geq 0$ implies

$$
\left.\nu(X)^{2}=\left(\int_{X} f^{\frac{1}{2}} f^{-\frac{1}{2}} d \nu\right)^{2} \leq \int_{X} f d \nu \int_{X} f^{-1} d \nu . \quad\right)
$$

Thus we have

Theorem 2.1. Let $A \subset \mathbb{R}^{2}$ be a Borel set and $\mu \in \mathcal{M}_{1}(A)$ with $I_{1}(\mu)<$ $\infty$. Then

$$
\int_{0}^{\pi} \mathcal{L}^{1}\left(p_{\theta}(A)\right) d \theta \geq \frac{\pi^{2}}{I_{1}(\mu)} .
$$

This gives immediately by the definition of $C_{1}$ (see (1.3)):

Corollary. $\int_{0}^{\pi} \mathcal{L}^{1}\left(p_{\theta}(A)\right) d \theta \geq \pi^{2} C_{1}(A)$.

This inequality is sharp with equality for discs. There is a higher dimensional generalization (see [M5]). For a Borel set $A \subset \mathbb{R}^{n}$,

$$
\int \mathcal{L}^{m}\left(P_{V}(A)\right) d \gamma_{n, m} V \geq c(n, m) C_{m}(A) .
$$

However, the sharp form of this inequality with equality for balls is known only in the cases $m=n-1$ and $m=n-2 \geq 1$. When $m=$ $n-2 \geq 1, C_{n-2}$ is the classical potential theoretic capacity in $\mathbb{R}^{n}$. Its relations to Lebesgue measure are well known. Combined with (2.2) this leads to the sharp inequality

$$
\int \mathcal{L}^{m}\left(P_{V}(A)\right) d \gamma_{n, m} V \geq b(n, m) \mathcal{L}^{n}(A)^{\frac{m}{n}}
$$

with equality for balls when $m=n-2$. This is also true for $m=1$ as proved by Chlebík by different methods (this is still unpublished). For other values of $m$ a sharp form of (2.3) is unknown.

It is quite easy to prove the sharp inequality

$$
\int \mathcal{L}^{n-1}\left(P_{V}(A)\right) d \gamma_{n, n-1} V \leq a(n) P(A)
$$


where $P(A)$ is the perimeter of $A$ (the $(n-1)$-area of $\partial A$, if $\partial A$ is sufficiently regular, but $P(A)$ can be defined for any Lebesgue measurable set in a distributional sense). Hence the proof of (2.3) for $m=n-1$ with equality for balls would sharpen the isoperimetric inequality. So far we only have this for $m=1, n=2$ by Chlebík's proof.

Baernstein and Loss have found in $[\mathbf{B L}]$ a connection of this problem to a conjecture about $m$-plane transforms.

We now explain how Theorem 2.1 can be used to get a lower bound in (2.1). This is very easy. Let

$$
\mu_{k}=c_{k} \mathcal{L}^{2} \mid U_{k}^{\frac{1}{4}} \text { with } c_{k}=\frac{1}{\mathcal{L}^{2}\left(U_{k}^{\frac{1}{4}}\right)} .
$$

Then easy estimates show

$$
I_{1}\left(\mu_{k}\right) \leq C k,
$$

whence

$$
\int_{0}^{\pi} \mathcal{L}^{1}\left(p_{\theta}\left(U_{k}^{\frac{1}{4}}\right)\right) d \theta \geq \frac{\pi^{2}}{C k}
$$

Peres and Solomyak in [PSo] showed that for some random $\frac{1}{4}$-Cantor sets $\frac{1}{k}$ is the correct asymptotic behaviour.

For $d<\frac{1}{4}$ similar methods give

$$
c d^{\left(1-s_{d}\right) k} \leq \int_{0}^{\pi} \mathcal{L}^{1}\left(p_{\theta}\left(U_{k}^{d}\right)\right) d \theta \leq \pi \sqrt{2} d^{\left(1-s_{d}\right) k},
$$

the upper bound is now trivial.

The same kind of arguments give more generally

Theorem 2.2. Let $0<s \leq m$ and $A \subset \mathbb{R}^{n}$ be a Borel set. If there exists $\mu \in \mathcal{M}_{1}(A)$ such that for some constant $b$,

$$
\mu(B(x, r)) \leq b r^{s} \text { for } x \in \mathbb{R}^{n}, \quad r>0,
$$

then for the $\varepsilon$-neighborhoods

$$
\begin{array}{cc}
A(\varepsilon)=\{x: \operatorname{dist}(x, A)<\varepsilon\}, \quad 0<\varepsilon<\frac{1}{2}, & \\
\int_{0}^{\pi} \mathcal{L}^{m}\left(P_{V}(A(\varepsilon))\right) d \gamma_{n, m} V \geq c(s) b^{-1} \varepsilon^{m-s}, & \text { if } 0<s<m,
\end{array}
$$

and

$$
\int_{0}^{\pi} \mathcal{L}^{m}\left(P_{V}(A(\varepsilon))\right) d \gamma_{n, m} V \geq c(m) b^{-1}\left(\log \left(\frac{1}{\varepsilon}\right)\right)^{-1}, \quad \text { if } s=m .
$$


The upper $s$-dimensional Minkowski content relative to $V$ is defined for $A \subset V$ by

$$
\mathcal{M}^{* s}(A)=\limsup _{\varepsilon \rightarrow 0} \alpha(m)^{-1} \varepsilon^{s-m} \mathcal{L}^{m}(A(\varepsilon) \cap V),
$$

where $\alpha(m)=\mathcal{L}^{m}(B(0,1))$.

Theorem 2.2 has an immediate corollary:

Corollary. If $0<s<m$ and $A \subset \mathbb{R}^{n}$ is a Borel set with $\mathcal{H}^{s}(A)>0$, then $\mathcal{M}^{* s}\left(P_{V}(A)\right)>0$ for $\gamma_{n, m}$ almost all $V \in G(n, m)$.

Peres, Simon and Solomyak have shown in [PSS1] that $\mathcal{M}^{* s}$ can be replaced by $s$-dimensional packing measure. As we learned in Section 1 it cannot be replaced by $\mathcal{H}^{s}$.

\section{Fourier transforms of measures with finite energy}

Now for a while we leave aside the projections and study Fourier transforms of measures. Later we shall return to their applications to projections.

Let $\mu$ be a finite Borel measure on $\mathbb{R}^{n}$. We define its Fourier transform by

$$
\hat{\mu}(x)=\int e^{-i x \cdot y} d \mu y, \quad x \in \mathbb{R}^{n} .
$$

The $s$-energy, $0<s<n$, of $\mu$ can be written as

$$
I_{s}(\mu)=\iint|x-y|^{-s} d \mu y d \mu x=\int k_{s} * \mu d \mu
$$

where $k_{s}$ is the Riesz kernel

$$
k_{s}(x)=|x|^{s-n} .
$$

We would now like to apply Plancherel's theorem, but we have to be careful since $k_{s}$ is in no $L^{p}$-space for $1 \leq p \leq \infty$. However, its Fourier transform exists in the distributional sense and is given by another Riesz kernel:

$$
\hat{k}_{s}(x)=b(s, n) k_{n-s}(x) .
$$

Formally by the convolution theorem and Plancherel's theorem we then have

$$
\begin{aligned}
I_{s}(\mu) & =\int\left(k_{s} * \mu\right) d \mu=c \int\left(k_{s} * \mu\right)^{\wedge} \overline{\hat{\mu}} d \mathcal{L}^{n} \\
& =c \int \hat{k}_{s} \hat{\mu} \hat{\mu} d \mathcal{L}^{n}=c \int \hat{k}_{s}|\hat{\mu}|^{2} d \mathcal{L}^{n} .
\end{aligned}
$$


(Here, and later, $c$ and $C$ may denote different constants at different occurrences.) This can be justified rigorously and we find

$$
I_{s}(\mu)=c(s, n) \int|x|^{s-n}|\hat{\mu}(x)|^{2} d x .
$$

How does the finiteness of $I_{s}(\mu)$ reflect on the behaviour of the Fourier transform of $\mu$, in particular, as $|x| \rightarrow \infty$ ? It is clear from (3.1) that if $I_{s}(\mu)<\infty$, then

$$
|\hat{\mu}(x)|<|x|^{-\frac{s}{2}}
$$

in a "big" set. But we don't have anything like this for all $x$ :

Example. Let $\mu$ be the standard Cantor measure, that is, $\mu$ is the restriction of $\mathcal{H}^{s}$ to the $\frac{1}{3}$-Cantor set in $\mathbb{R}$ where $s=\frac{\log 2}{\log 3}$. Then

$$
I_{t}(\mu)<\infty \text { for all } 0<t<s .
$$

The Fourier transform of $\mu$ is given by

$$
\hat{\mu}(x)=\prod_{k=1}^{\infty} \cos \left(3^{-k} x\right) \text { for } x \in \mathbb{R} .
$$

It is then clear that $\hat{\mu}\left(3^{j} \pi\right) \nrightarrow 0$ as $j \rightarrow \infty$.

For other self-similar Cantor sets with dissection ratio $d$ in place of $\frac{1}{3}$, it depends on the number theoretic properties of $d$ whether or not the Fourier transform of the corresponding measure tends to zero at infinity; it is not a question of size. See $[\mathbf{K S}]$ for these and many related things.

The above considerations motivate the following definition:

Definition. The Fourier dimension of $A \subset \mathbb{R}^{n}$ is

$\operatorname{dim}_{F} A=\sup \{s \in[0, n]: \exists \mu \in \mathcal{M}(A)$ such that

$$
\left.|\hat{\mu}(x)| \leq|x|^{-\frac{s}{2}} \text { for } x \in \mathbb{R}^{n}\right\} .
$$

It is an easy exercise to derive from (1.2) and (3.1) that

$$
\operatorname{dim}_{F} A \leq \operatorname{dim} A
$$

for all Borel sets $A \subset \mathbb{R}^{n}$.

The above example suggests that $\operatorname{dim}_{F} C=0$ for the $\frac{1}{3}$-Cantor set $C$. To really prove this, one has to show that all Borel measures on $C$ behave like the Cantor measure. And this is true: for every $\mu \in \mathcal{M}(C), \hat{\mu}(x) \nrightarrow 0$ as $x \rightarrow \infty$.

Another simple example with zero Fourier dimension is the boundary of any cube in $\mathbb{R}^{n}$. This is very easy to see. For example in the plane this 
is essentially the same as saying that $\operatorname{dim}_{F} I=0$ for the segment $I=$ $\{(x, 0): 0 \leq x \leq 1\}$. But if $\mu \in \mathcal{M}(I)$, then

$$
\hat{\mu}(u, v)=\hat{\mu}(u, 0) \text { for }(u, v) \in \mathbb{R}^{2},
$$

whence $\hat{\mu}(u, v) \nrightarrow 0$ as $v \rightarrow \infty$ whenever $\hat{\mu}(u, 0) \neq 0$. Note, however, that the Fourier dimension of $[0,1]$ in $\mathbb{R}$ is 1 . So the Fourier dimension depends on the space where the set lives.

Let $\sigma$ be the surface measure on $S^{n-1}$ (i.e., $\mathcal{H}^{n-1} \mid S^{n-1}$ normalized in any way you like). Then, by a classical formula,

$$
\hat{\sigma}(x)=c|x|^{-\frac{(n-2)}{2}} J_{\frac{(n-2)}{2}}(|x|),
$$

where $J_{\alpha}$ denotes the Bessel function of order $\alpha$. A basic estimate for Bessel functions is

$$
\left|J_{\alpha}(t)\right| \leq C t^{-\frac{1}{2}} \text { for } t>0 \text {. }
$$

Thus

$$
|\hat{\sigma}(x)| \leq C|x|^{-\frac{(n-1)}{2}} \text { for } x \in \mathbb{R}^{n} .
$$

By (3.2), this gives

$$
\operatorname{dim}_{F} S^{n-1}=n-1=\operatorname{dim} S^{n-1} .
$$

Hence $S^{n-1}$ is an example of a Salem set:

Definition. We say that a Borel set $A \subset \mathbb{R}^{n}$ is a Salem set if $\operatorname{dim}_{F} A=$ $\operatorname{dim} A$.

So roughly speaking, for $A$ to be a Salem set means that we can put a measure on it whose Fourier transform decays at infinity as quickly as its Hausdorff dimension allows.

The first Salem sets of Salem were random sets. Later it turned out that Salem sets abound as random sets. The trajectories and level sets of Brownian motion and many others are Salem sets. See the book of Kahane $[\mathbf{K}]$.

Deterministic Salem sets of non-integral dimension are hard to find. The first ones for any given $0<s<1$ were constructed by Kaufman in $[\mathbf{K 2}]$. It is a number theoretic set of real numbers satisfying certain diophantine approximation conditions. A recent and more geometric example was given by Bluhm in $[\mathbf{B}]$.

Very little seems to be known about the possible properties of Salem sets or the related "Salem measures". Mitsis asked in [Mi2] the following interesting question: for which values of $s, 0<s<n$, is there a $\mu \in$ $\mathcal{M}\left(\mathbb{R}^{n}\right)$ such that

$$
\frac{r^{s}}{c} \leq \mu(B(x, r)) \leq C r^{s} \text { for } x \in \operatorname{spt} \mu, \quad 0<r<1,
$$


and

$$
|\hat{\mu}(x)| \leq|x|^{-\frac{s}{2}} \text { for } x \in \mathbb{R}^{n} .
$$

No such a measure is known for any non-integral $s$.

These considerations should convince the reader that the decay of Fourier transforms of measures with finite energy is a delicate matter. Let us try to see how much more we can say. First, if $I_{s}(\mu)<\infty$ it is almost trivial that

$$
\int_{B(0, R)}|\hat{\mu}(x)|^{2} d \mathcal{L}^{n} x \leq C R^{n-s}
$$

and

$$
R^{s-n} \int_{B(0, R)}|\hat{\mu}(x)|^{2} d x \leq \int_{B(0, R)}|x|^{s-n}|\hat{\mu}(x)|^{2} d x \leq C I_{s}(\mu) .
$$

A partial converse is also true: if (3.3) holds, then $I_{t}(\mu)<\infty$ for $t<s$. This can be easily seen by writing the integral $\int_{\mathbb{R}^{n} \backslash B(0,1)}|x|^{t-n}|\hat{\mu}(x)|^{2} d x$ as the sum over spherical rings $B\left(0,2^{j}\right) \backslash B\left(0,2^{j-1}\right), j=1,2, \ldots$.

Let us now look at averages over smaller sets, namely the spheres $S(r)=\left\{y \in \mathbb{R}^{n}:|y|=r\right\}$. Denote them by

$$
\sigma(\mu)(r)=\int|\hat{\mu}|^{2} d \tau_{r}=c \int_{S^{n-1}}|\hat{\mu}(r \zeta)|^{2} d \zeta
$$

where $\tau_{r}$ denotes the normalized $\left(\tau_{r}\left(S^{n-1}(r)\right)=1\right)$ area measure on $S(r)$. The Fourier transform of $\tau_{r}$ is given by

$$
\hat{\tau}_{r}(x)=c(r|x|)^{-\frac{(n-2)}{2}} J_{\frac{(n-2)}{2}}(r|x|) .
$$

Hence we have by the convolution formula and the Plancherel formula (note that $\left(\hat{\tau}_{r}\right)^{\wedge}=\tau_{r}$ )

$$
\begin{aligned}
\sigma(\mu)(r) & =\int\left(\mu * \hat{\tau}_{r}\right) d \mu \\
& =c \iint(r|x-y|)^{-\frac{(n-2)}{2}} J_{\frac{(n-2)}{2}}(r|x-y|) d \mu y d \mu x
\end{aligned}
$$

Using again the basic estimate $\left|J_{\alpha}(t)\right| \leq C t^{-\frac{1}{2}}$ for Bessel functions, we get

$$
\sigma(\mu)(r) \leq C r^{-\frac{(n-1)}{2}} I_{\frac{(n-1)}{2}}(\mu) .
$$

Of course, this means something only if $I_{\frac{(n-1)}{2}}(\mu)<\infty$. And this is then the best possible decay that we can hope for from this information. 
Namely,

$$
I_{s}(\mu)=c \int|x|^{s-n}|\hat{\mu}(x)|^{2} d x=c \int_{0}^{\infty} r^{s-1} \sigma(\mu)(r) d r .
$$

Thus if $\sigma(\mu)(r) \leq c r^{-t}$ for $r>0$, then $I_{s}(\mu)<\infty$ for all $s<t$.

It is not very difficult to extend this to $0<s \leq \frac{(n-1)}{2}$, see [M4];

$$
\sigma(\mu)(r) \leq c r^{-s} I_{s}(\mu) \text { for } r>0, \quad 0<s \leq \frac{(n-1)}{2} .
$$

But this does not extend beyond $\frac{(n-1)}{2}$. An example in the plane can be given as follows. Let $\frac{1}{2}<s<1$ and let $\mu$ be a Borel probability measure in $\{(x, 0): 0 \leq x \leq 1\} \subset \mathbb{R}^{2}$ such that $I_{s}(\mu)<\infty$ and $\hat{\mu}(x, 0) \nrightarrow 0$ as $x \rightarrow \infty$. They exist for all such $s$. Since $\hat{\mu}$ is Lipschitz continuous, we can find $\delta, \eta>0$ and arbitrarily large radii $r$ such that

$$
|\hat{\mu}(x, 0)|>\eta \text { for } r-\delta<x<r+\delta .
$$

Now $\hat{\mu}(x, y)=\hat{\mu}(x, 0)$ directly by the definition of $\hat{\mu}$ and the fact that spt $\mu \subset\{(x, 0): x \in \mathbb{R}\}$. By elementary geometry it then follows that

$$
|\hat{\mu}(x, y)|>\eta
$$

when $(x, y)$ belongs to a subarc of $\{\zeta:|\zeta|=r\}$ with length comparable to $\sqrt{r}$. This implies that

$$
\sigma(\mu)(r) \geq C r^{-\frac{1}{2}} .
$$

Hence for $\frac{1}{2}<s<1$ we cannot improve the decay given by (3.5). Other examples and partial results in the range of $\frac{(n-1)}{2}<s<n$ are given in $[\mathbf{M} 4]$ and $[\mathbf{S 1}]$.

Let us denote by $\alpha(s)$ the best possible rate of decay at infinity which the condition $I_{s}(\mu)<\infty$ implies for the averages $\sigma(\mu)(r)$. More precisely,

$$
\begin{aligned}
\alpha(s)=\sup \left\{\alpha: \sigma(\mu)(r) \leq C r^{-\alpha} \text { for } r\right. & >0, \\
\mu & \left.\in \mathcal{M}_{1}\left(\mathbb{R}^{n}\right) \text { with } I_{s}(\mu)<\infty\right\} .
\end{aligned}
$$

The discussion above shows that $\alpha(s)=s$ for $0<s \leq \frac{(n-1)}{2}$, and $\alpha(s)=\frac{1}{2}$ for $\frac{1}{2}<s<1$ and $n=2$. Examples of Sjölin in [S1] also show that $\alpha(s)=\frac{(n-1)}{2}$ for $\frac{(n-1)}{2}<s<\frac{n}{2}$ in $\mathbb{R}^{n}$. Other information in terms of examples and partial results can be found in [M4], [S1] and [S2]. In general the precise value of $\alpha(s)$ is unknown but Wolff solved this problem completely in the plane in [W2]: 
Theorem 3.1. For $n=2$,

$$
\alpha(s)= \begin{cases}s & \text { for } 0<s \leq \frac{1}{2}, \\ \frac{1}{2} & \text { for } \frac{1}{2}<s \leq 1, \\ \frac{s}{2} & \text { for } 1<s<2 .\end{cases}
$$

The proof is very hard. It is based on geometric "Kakeya-type" arguments which many people including Fefferman, Córdoba, Bourgain and Wolff have developed since the beginning of the 1970's in connection with various questions in Fourier analysis. We return to this a little more in the next section.

Let us now study a particular geometric problem and see how the Fourier transform can be applied to it. We define the distance set of $A \subset$ $\mathbb{R}^{n}$ as

$$
D(A)=\{|x-y|: x, y \in A\} \subset[0, \infty) .
$$

The question is: if $A$ is big, is $D(A)$ also big? One answer is the classical Steinhaus theorem which tells us that if $A$ is Lebesgue measurable and $\mathcal{L}^{n}(A)>0$, then $D(A)$ contains some interval $[0, \varepsilon), \varepsilon>0$. But here we mean by "big" a lower bound on the Hausdorff dimension. The following result was proved by Falconer in [F3]:

Theorem 3.2. If $A \subset \mathbb{R}^{n}$ is a Borel set with $\operatorname{dim} A>\frac{(n+1)}{2}$, then $\mathcal{L}^{1}(D(A))>0$.

Proof: By (1.2) there is $\mu \in \mathcal{M}(A)$ such that $I_{(n+1) / 2}(\mu)<\infty$. Let us define a "distance measure" $\delta(\mu)$ related to $\mu$ by taking the image of $\mu \times \mu$ under the distance map. In other words,

$$
\delta(\mu)(B)=\int \mu(\{y:|x-y| \in B\}) d \mu x
$$

for Borel sets $B \subset \mathbb{R}$. Then $\delta(\mu) \in \mathcal{M}(D(A))$.

Let $0<\varepsilon<r$ and let $\chi_{r, \varepsilon}$ be the characteristic function of the ring $\left\{z \in \mathbb{R}^{n}: r<|z|<r+\varepsilon\right\}$. Then

$$
\delta(\mu)((r, r+\varepsilon))=\int \chi_{r, \varepsilon} * \mu d \mu=c \int \hat{\chi}_{r, \varepsilon}|\hat{\mu}|^{2} d \mathcal{L}^{n}
$$

by the convolution and Plancherel theorems. The formula for the Fourier transform of a radial function gives

$$
\hat{\chi}_{r, \varepsilon}(x)=c|x|^{\frac{(2-n)}{2}} \int_{r}^{r+\varepsilon} J_{\frac{(n-2)}{2}}(u|x|) u^{\frac{n}{2}} d u .
$$


Estimating again the Bessel function by $C(u|x|)^{-\frac{1}{2}}$, we get, since $\varepsilon<r$,

$$
\left|\hat{\chi}_{r, r+\varepsilon}(x)\right| \leq C r^{\frac{(n-1)}{2}}|x|^{\frac{(1-n)}{2}} \varepsilon .
$$

Substituting this into (3.6) we find

$$
\begin{aligned}
\delta(\mu)((r, r+\varepsilon)) & \leq C r^{\frac{(n-1)}{2}} \varepsilon \int|x|^{\frac{(1-n)}{2}}|\hat{\mu}(x)|^{2} d \mathcal{L}^{n} x \\
& =C r^{\frac{(n-1)}{2}} I_{\frac{(n+1)}{2}}(\mu) \varepsilon
\end{aligned}
$$

for $0<\varepsilon<r$. Clearly this estimate implies that $\delta(\mu) \ll \mathcal{L}^{1}$, even with $L^{\infty}$ Radon-Nikodým derivative. As spt $\delta(\mu) \subset D(A)$, we get $\mathcal{L}^{1}(D(A))>$ 0 .

Although the above proof with Fourier transform is quite simple, no proof without it is known.

As we shall see below, the bound $\frac{(n+1)}{2}$ is not sharp, at least for $n=$ 2,3 . It is generally believed that $\operatorname{dim} A>\frac{n}{2}$ should imply $\mathcal{L}^{1}(D(A))>0$ when $n \geq 2$. For $n=1, \mathcal{L}^{1}(D(A))=0$ for some $A$ with $\operatorname{dim} A=1$. Simple examples show that one cannot do better than $\frac{n}{2}$.

The conclusion $\delta(\mu) \in L^{\infty}$ in the above proof gives a clear hope for improvement: it should be possible to get $\delta(\mu) \in L^{2}$ with weaker assumptions. The first such improvement was done by Bourgain in [B2] for $n=2$ and $n=3$. The best result in $\mathbb{R}^{2}$ is now due to Wolff based on Theorem 3.1:

Theorem 3.3. If $A \subset \mathbb{R}^{2}$ is a Borel set with $\operatorname{dim} A>\frac{4}{3}$, then $\mathcal{L}^{1}(D(A))>$ 0 .

The proof is based on a closer look at the distance measures $\delta(\mu)$. Let us first see how they look in $\mathbb{R}^{n}$. For more details, see [M4]. Let $\mu \in$ $\mathcal{M}\left(\mathbb{R}^{n}\right)$ with $I_{s}(\mu)<\infty$ for some $s>0$. Suppose first that $\mu \ll \mathcal{L}^{n}$ with smooth Radon-Nikodým derivative. Then $\delta(\mu) \ll \mathcal{L}^{1}$ and (identifying it with its Radon-Nikodým derivative) one can derive a formula for it from (3.6) and (3.7):

$$
\delta(\mu)(u)=c u^{\frac{n}{2}} \int_{0}^{\infty} r^{\frac{n}{2}} J_{\frac{(n-2)}{2}}(r u) \sigma(\mu)(r) d r .
$$

Thus $\delta(\mu)$ is a kind of transform (a Hankel transform) of $\sigma(\mu)$, and there is a Plancherel theorem for it which gives

$$
\int_{0}^{\infty} u^{1-n} \delta(\mu)(u)^{2} d u=c \int_{0}^{\infty} r^{n-1} \sigma(\mu)(r)^{2} d r .
$$


The rest of the strategy is to estimate one factor $\sigma(\mu)(r)$ in $\sigma(\mu)(r)^{2}$ by $r^{-\alpha} \sigma(\mu)(r)$ where $\alpha<\alpha(s)$. Unfortunately, we only have an estimate good enough for this to be useful when $n=2$. Let us now see how this works in the proof of Theorem 3.3.

We still assume that $\mu$ is a smooth non-negative function with compact support. Let $s>\frac{4}{3}$ and $\frac{4}{3}<t<s$. A quantitative version of Theorem 3.1 says that

$$
\sigma(\mu)(r) \leq C r^{-\frac{t}{2}} I_{s}(\mu) \text { for } r>0,
$$

and for $\mu \in \mathcal{M}(B(0,1))$; a restriction to $B(0,1)$ or something like it is needed for a quantitative estimate, but it is no harm for us. Here $C$ depends on $s$ and $t$ but not on $\mu$. Putting this into (3.10) we get by (3.4) (with $n=2$ ),

$$
\begin{aligned}
\int_{0}^{\infty} u^{-1} \delta(\mu)(u)^{2} d u & \leq C \int_{0}^{\infty} r \sigma(\mu)(r) r^{-\frac{t}{2}} d r I_{s}(\mu) \\
& =C \int_{0}^{\infty} r^{\left(2-\frac{t}{2}\right)-1} \sigma(\mu)(r) d r I_{s}(\mu)=C I_{2-\frac{t}{2}}(\mu) I_{s}(\mu) \\
& \leq C I_{s}(\mu)^{2}
\end{aligned}
$$

where the last inequality comes from the facts that $2-\frac{t}{2}<t<s$ (since $\frac{4}{3}<t<s$ ) and that $\operatorname{spt} \mu \subset B(0,1)$. This inequality remains valid for any $\mu \in \mathcal{M}_{1}(B(0,1))$ with $I_{s}(\mu)<\infty$ by approximation by smooth functions. In particular, it shows that $\delta(\mu) \ll \mathcal{L}^{1}$ if $I_{s}(\mu)<$ $\infty$ with $s>\frac{4}{3}$. This completes the proof of Theorem 3.3 since the assumption $\operatorname{dim} A>\frac{4}{3}$ allows us to find such a $\mu \in \mathcal{M}_{1}(A)$.

Let us make a few comments on the above proofs of Theorems 3.2 and 3.3. In both the key idea was to show that the finiteness of a certain energy of $\mu$ forces $\delta(\mu)$ to be a function in $L^{p}$ with $p=\infty$ or $p=2$. For such a method with $p>1$ it is always enough to prove uniform estimates with smooth functions, and one also needs estimates for $\sigma(\mu)$ such as (3.5) only in the case of smooth functions $\mu$. But it is essential that they are non-negative; obviously (3.5) cannot hold for all smooth functions $\mu$ with support in $B(0,1)$.

In the case $s>\frac{(n+1)}{2}$, Sjölin and I showed in $[\mathbf{M S}]$ that $\delta(\mu)$ is continuous if $I_{s}(\mu)<\infty$. This gives an improvement of Theorem 3.2:

Theorem 3.4. If $A \subset \mathbb{R}^{n}$ is a Borel set with $\operatorname{dim} A>\frac{(n+1)}{2}$, then $D(A)$ contains some non-empty open interval.

Simple examples show that it need not contain any such interval with 0 as an end-point. It is not known, even when $n=2$, whether $\frac{(n+1)}{2}$ 
could be improved in Theorem 3.4. However there is a simple example in [M4] showing that if $s<\frac{(n+1)}{2}, \delta(\mu)$ need not be bounded even when $I_{s}(\mu)<\infty$.

Below the level where we don't know anymore that $\mathcal{L}^{1}(D(A))>0$ we can still say something about $\operatorname{dim} D(A)$. Falconer proved in $[\mathbf{F 3}]$ that

$$
\operatorname{dim} D(A) \geq \operatorname{dim} A-\frac{(n-1)}{2} \quad \text { when } \quad \frac{(n-1)}{2} \leq \operatorname{dim} A \leq \frac{(n+1)}{2} .
$$

Wolff's result Theorem 3.1 improves this in $\mathbb{R}^{2}$ :

$$
\operatorname{dim} D(A) \geq \frac{3}{2} \operatorname{dim} A-1 \text { when } 1 \leq \operatorname{dim} A \leq \frac{4}{3} \text { and } n=2 .
$$

Mitsis proved in $[\mathbf{M i 1}]$ that if $A \subset \mathbb{R}^{2}$ with $\mathcal{H}^{s}(A)>0$, where $\frac{1}{2}<$ $s<1$, then $\mathcal{H}^{s-\frac{1}{2}}(D(A))>0$. This (and a slightly more general version in $[\mathrm{Mi1}])$ is the only non-trivial result about distance sets in this spirit that I know whose proof does not use Fourier transforms.

In [W3] Wolff studied the $L^{1}$ averages of $\sigma(\mu)$ and showed that they are connected with the so-called Furstenberg problem. In $[\mathbf{K T}]$ Katz and Tao showed that certain discrete versions of the distance set problem, Furstenberg problem and Erds ring problem are equivalent. The Erds ring problem was recently solved by Edgar and Miller in $[\mathbf{E M}]$. They showed that if $R \subset \mathbb{R}$ is a Borel set and a subring of $\mathbb{R}$, then either $R=\mathbb{R}$ or $\operatorname{dim} R=0$. We shall not discuss these interesting problems here.

Sjlin and Soria have investigated in $[\mathbf{S 2}],[\mathbf{S 3}],[\mathbf{S S 1}]$ and $[\mathbf{S S 2}]$ the averages over different sets and measures in place of the surface measures on spheres, and for different classes of functions in place of the nonnegative functions.

\section{Besicovitch sets}

Here we discuss some geometric measure theoretic problems whose relations to Fourier analysis have been under active interest. We shall discuss these relations only briefly and we recommend the reader to consult the books $[\mathbf{G}]$ of de Guzmán and [F2] of Falconer, the survey articles $[\mathbf{T a}]$ of Tao and $[\mathbf{W} \mathbf{1}]$ of Wolff and the lecture notes $[\mathbf{M i 4}]$ of Mitsis. As we shall see, this topic is also naturally related to orthogonal projections.

In 1919, Besicovitch constructed a strange set; a set $B \subset \mathbb{R}^{2}$ of Lebesgue measure zero that contains a unit line segment in every direction. See $[\mathbf{F 2}]$ or $[\mathbf{G}]$ for how to perform such constructions and for the history of this question. In 1964 Besicovitch observed that this 
could be obtained in a very elegant manner from a theorem that he had proven almost 30 years earlier, and which was mentioned in Section 1: a purely unrectifiable set with finite $\mathcal{H}^{1}$ measure projects into zero length in almost all directions. However, we need this information only for the Cantor set $C_{\frac{1}{4}}$ for which we gave a direct proof in Section 2. We now show how to get Besicovitch's set in this manner.

Theorem 4.1. There exists a Borel set $B \subset \mathbb{R}^{2}$ such that $\mathcal{L}^{2}(B)=0$ and $B$ contains a whole line in every direction.

Proof: Let $C \subset \mathbb{R}^{2}$ be a compact set such that $\pi(C)=[0,1]$, where $\pi(x, y)=x$ for $(x, y) \in \mathbb{R}^{2}$, and $\mathcal{L}^{1}\left(p_{\theta}(C)\right)=0$ for $\mathcal{L}^{1}$ almost all $\theta \in$ $[0, \pi)$.

We can take as $C$ a suitably rotated and dilated copy of $C_{\frac{1}{4}}$ or we can modify the construction of $C_{\frac{1}{4}}$ by placing the first four disjoint closed squares of side-length $\frac{1}{4}$ inside $[0,1] \times[0,1]$ so that their projections cover $[0,1]$. Consider the lines

$$
\ell(a, b)=\{(x, y): y=a x+b\}, \quad(a, b) \in C,
$$

and define $B$ as their union:

$$
B=\bigcup_{(a, b) \in C} \ell(a, b)=\{(x, a x+b): x \in \mathbb{R},(a, b) \in C\} .
$$

From the latter presentation it is easy to see that $B$ is $\sigma$-compact and whence a Borel set. Since $\pi(C)=[0,1], B$ contains a line $\ell(a, b)$ for all $0 \leq a \leq 1$. Taking a union of four rotated copies of $B$ we get a Borel set that contains a line in every direction. It remains to show that $\mathcal{L}^{2}(B)=0$.

We do this by showing that almost every vertical line meets $B$ in a set of length zero and then using Fubini's theorem. For any $t \in \mathbb{R}$,

$$
\begin{aligned}
B \cap\{(x, y): x=t\} & =\{(t, a t+b):(a, b) \in C\} \\
& =\{t\} \times \pi_{t}(C),
\end{aligned}
$$

where $\pi_{t}(x, y)=t x+y$. The map $\pi_{t}$ is essentially a projection $p_{\theta}$ for some $\theta$, and hence we have $\mathcal{L}^{1}\left(\pi_{t}(C)\right)=0$ for $\mathcal{L}^{1}$ almost all $t \in \mathbb{R}$. Thus $\mathcal{L}^{2}(B)=0$.

We now use the projection theorems of Section 1 to prove that such a set $B$ must have Hausdorff dimension 2. This result is due to Davies, see $[\mathbf{F 2}]$. We use the same notation $\pi$ and $\pi_{t}$ as in the above proof.

Theorem 4.2. Let $B \subset \mathbb{R}^{2}$ contain a line in every direction. Then $\operatorname{dim} B=2$. 
Proof: We can assume that $B$ is a Borel set since every set in $\mathbb{R}^{2}$ is contained in a $G_{\delta}$-set of the same dimension. Let $C$ be the set of $(a, b)$ such that $\ell(a, b) \subset B$. Then $C$ is again a Borel set. Since for every $a \in \mathbb{R}$, some $\ell(a, b) \subset B$, we have $\pi(C)=\mathbb{R}$, and so $\mathcal{H}^{1}(C)=\infty$. By Theorem 1.1(1), for almost $t \in \mathbb{R}, \operatorname{dim} \pi_{t}(C)=1$.

Looking at (4.1) we see that this means that almost all vertical sections of $B$ have dimension 1. A rather easy Fubini-type inequality for Hausdorff measures, e.g., [F2, p. 72] or [M6, p. 104], then implies that $\operatorname{dim} B=2$.

If $B \subset \mathbb{R}^{2}$ is as in Theorem 4.1, then $A=B \times \mathbb{R}^{n-2}$ is a Borel set in $\mathbb{R}^{n}$ with $\mathcal{L}^{n}(A)=0$ and containing a line in every direction. The above proof of Theorem 4.2 does not generalize to $\mathbb{R}^{n}$ and it is an open problem whether any such set $A$ has to have Hausdorff dimension $n$. Lower bounds have been obtained by Bourgain, Wolff, and Katz, Laba and Tao, see $[\mathbf{B} \mathbf{1}],[\mathbf{W} \mathbf{1}],[\mathbf{L T}]$ and the references given there.

To illustrate quickly why such Besicovitch sets are interesting from the point of view of Fourier analysis, we mention one connection: if there is a Borel set $B \subset \mathbb{R}^{n}$ with $\operatorname{dim} B<n$ containing a line in every direction, then Stein's restriction conjecture is false. This conjecture (or one form of it) says that the Fourier transform restricted to $S^{n-1}$ defines a bounded operator $L^{\infty}\left(S^{n-1}\right) \rightarrow L^{p}\left(\mathbb{R}^{n}\right)$ for $p>\frac{2 n}{(n-1)}$. For $n=2$ this is known to be true, but so is Theorem 4.2.

Another interesting open question is: for which pairs of integers $(m, n)$, $0<m<n$, are there Borel sets $B \subset \mathbb{R}^{n}$ such that $\mathcal{L}^{n}(B)=0$ and $B$ contains an $m$-plane in every direction? We know that they exist for $(1, n)$ for all $n$. They don't exist for $(m, n)$ when $m>\frac{n}{2}$ by results of Marstrand and Falconer. We sketch a proof of this soon. We shall not discuss here the much harder improvements by Bourgain, see [B1]. In [Mi3] Mitsis showed that if $A \subset \mathbb{R}^{n}$ contains a 2-plane in every direction then $\operatorname{dim} A=n$.

We return now to orthogonal projections. We know that for a Borel set $A \subset \mathbb{R}^{n}$, if $\operatorname{dim} A>m$, then $\mathcal{L}^{m}\left(P_{V}(A)\right)>0$ for $\gamma_{n, m}$ almost all $V \in G(n, m)$. But can we say more? Does $P_{V}(A)$ have interior points in $V$ ? If not, does it if $\operatorname{dim} A$ is sufficiently big? In the plane the answer is no:

Example 4.3. There is a Borel set $A \subset \mathbb{R}^{2}$ such that $\operatorname{dim} A=2$, and even $\mathcal{L}^{2}\left(\mathbb{R}^{2} \backslash A\right)=0$, but the interior of $p_{\theta}(A)$, Int $p_{\theta}(A)$, is empty for all $\theta \in[0, \pi)$. 
Proof: Let $B$ be the Besicovitch set of Theorem 4.1 and

$$
A=\mathbb{R}^{2} \backslash \bigcup_{q \in \mathbb{Q}^{2}}(B+q) .
$$

Then $A$ has all the required properties.

When we go to higher dimensions the situation changes as has been shown by Peres and Schlag in $[\mathbf{P S}]$. We shall discuss their interesting work more in the next two sections, but we introduce now one of the basic notions for it; the Sobolev norm of a locally finite Borel measure $\nu$. It is defined for $\gamma \in \mathbb{R}$ by

$$
\|\nu\|_{2, \gamma}=\left(\int_{\mathbb{R}^{n}}|x|^{2 \gamma}|\hat{\nu}(x)|^{2} d x\right)^{\frac{1}{2}} .
$$

Note that \|\|$_{2,0}$ is just the $L^{2}$-norm, so $\|\nu\|_{2,0}<\infty$ implies that $\nu \in L^{2}$, that is, $\nu \ll \mathcal{L}^{2}$ with Radon-Nikodým derivative in $L^{2}$.

The larger the $\gamma$ for which $\|\nu\|_{2, \gamma}<\infty$, the more regularity $\nu$ has. One says often that if $\gamma>0$ and $\|\nu\|_{2, \gamma}<\infty$, then $\nu$ has $\gamma$ fractional derivatives in $L^{2}$ (though $\gamma$ need not be an integer). This is of course motivated by the formula $\left(\partial_{j} f\right)^{\wedge}(x)=x_{j} \hat{f}(x)$, which yields that \|\|$_{2, \gamma}$ really defines the norm in the classical Sobolev space $W^{2, \gamma}$ when $\gamma$ is an integer. We have then

Proposition 4.4. If $\nu \in \mathcal{M}\left(\mathbb{R}^{n}\right)$ and $\|\nu\|_{2, \gamma}<\infty$ for some $\gamma$ with $2 \gamma>n$, then $\nu$ is a continuous function.

Proof: By Schwartz's inequality

$$
\begin{aligned}
\int|\hat{\nu}(x)| d x \leq( & \left.(1+|x|)^{2 \gamma}|\hat{\nu}(x)|^{2} d x\right)^{\frac{1}{2}}\left(\int(1+|x|)^{-2 \gamma} d x\right)^{\frac{1}{2}} \\
\leq & \left(\int_{B(0,1)}|\hat{\nu}(x)|^{2} d x+\int|x|^{2 \gamma}|\hat{\nu}(x)|^{2} d x\right)<\infty .
\end{aligned}
$$

Thus $\hat{\nu} \in L^{1}$ and so $\nu$ is continuous.

Let us look now at the Sobolev norms of the one-dimensional projections of a measure $\mu \in \mathcal{M}\left(\mathbb{R}^{n}\right)$. We parametrize them by the unit sphere and set

$$
\begin{gathered}
p_{\theta} x=\theta \cdot x, \quad x \in \mathbb{R}^{n}, \quad \theta \in S^{n-1}, \\
\left(p_{\theta} \mu\right)(B)=\mu\left(p_{\theta}^{-1}(B)\right) .
\end{gathered}
$$


Theorem 4.5. Let $\mu \in \mathcal{M}\left(\mathbb{R}^{n}\right)$ and let $0<1+2 \gamma<n$. Then

$$
\int_{S^{n-1}}\left\|p_{\theta} \mu\right\|_{2, \gamma}^{2} d \theta=C I_{1+2 \gamma}(\mu) .
$$

Proof: Clearly for $\theta \in S^{n-1}, t \in \mathbb{R}$,

$$
\widehat{p_{\theta} \mu}(t)=\hat{\mu}(t \theta) \text {. }
$$

Using Plancherel's theorem and (3.1) we have

$$
\begin{aligned}
\int_{S^{n-1}}\left\|p_{\theta} \mu\right\|_{2, \gamma}^{2} d \theta & =c \int_{S^{n-1}} \int_{-\infty}^{\infty}\left|\widehat{p_{\theta} \mu}(t)\right|^{2}|t|^{2 \gamma} d t d \theta \\
& =c \int_{S^{n-1}} \int_{-\infty}^{\infty}|\hat{\mu}(t \theta)|^{2}|t|^{2 \gamma} d t d \theta \\
& =2 c \int_{\mathbb{R}^{n}}|\hat{\mu}(x)|^{2}|x|^{1+2 \gamma-n} d x \\
& =c I_{1+2 \gamma}(\mu) .
\end{aligned}
$$

Combining Proposition 4.4 with Theorem 4.5 we obtain easily

Theorem 4.6. If $A \subset \mathbb{R}^{n}$ is a Borel set with $\operatorname{dim} A>2$, then $\operatorname{Int} p_{\theta}(A) \neq$ $\emptyset$ for almost all $\theta \in S^{n-1}$.

Proof: Let $\mu \in \mathcal{M}_{1}(A)$ with $I_{s}(\mu)<\infty$ for some $s>2$. Defining $\gamma$ by $1+2 \gamma=s$, we have $2 \gamma>1,\left\|p_{\theta} \mu\right\|_{2, \gamma}<\infty$ for almost $\theta \in S^{n-1}$ by Theorem 4.5, whence $p_{\theta} \mu$ is a continuous function by Proposition 4.4. As spt $p_{\theta} \mu \subset p_{\theta}(A)$, we get Int $p_{\theta}(A) \neq \emptyset$ for almost $\theta \in S^{n-1}$.

We can consider the set $A$ of Example 4.3 since $A \subset \mathbb{R}^{2} \subset \mathbb{R}^{n}$ to see that the lower bound 2 in Theorem 4.6 is sharp. Similar arguments yield that if $A \subset \mathbb{R}^{n}$ is a Borel set with $\operatorname{dim} A>2 m$, then $\operatorname{Int} P_{V}(A) \neq \emptyset$ for almost all $V \in G(n, m)$. Here the lower bound $2 m$ may not be sharp.

Above we used Besicovitch sets in $\mathbb{R}^{2}$, to show that $\operatorname{Int} p_{\theta}(A)$ may be empty for all $\theta$ despite $\operatorname{dim} A=2$. We can reverse this argument to show that if $A \subset \mathbb{R}^{3}$ contains a plane in every direction, then $\mathcal{L}^{3}(A)>0$. For if not, we could argue exactly as in Example 4.3 to get a contradiction with Theorem 4.6. Similarly the higher dimensional version of Theorem 4.6 mentioned above implies that if $2 m>n$ and $A \subset \mathbb{R}^{n}$ contains an $m$-plane in every direction, then $\mathcal{L}^{n}(A)>0$. 


\section{Generalized projections of Peres and Schlag}

In Section 1 we proved dimension results for orthogonal projections $p_{\theta}$, $\theta \in[0, \pi)$. Examining the proofs, one quickly notices that they could be generalized to some kind of an axiomatic setting. The key point was that we had a parametrized family of mappings $p_{\theta}$ and a measure $\lambda$ on the parameter interval such that

$$
\lambda\left(\left\{\theta:\left|p_{\theta}(x)-p_{\theta}(y)\right| \leq t\right\}\right) \leq \frac{C t}{|x-y|} .
$$

But to make such a generalization really useful, one needs good new tools and ideas. These are provided by effective use of the Sobolev norm introduced in the previous section and its Littlewood-Paley decomposition.

Here we first present the basic general theorems with some comments on the proofs. Then we give some applications. For many more details and applications the reader is recommended to consult the paper [PS] of Peres and Schlag.

Recall that the Sobolev norm of $\nu \in \mathcal{M}\left(\mathbb{R}^{n}\right)$ is defined by

$$
\|\nu\|_{2, \gamma}^{2}=\int|x|^{2 \gamma}|\hat{\nu}(x)|^{2} d x,
$$

where $\gamma \in \mathbb{R}$. Related to it we define the Sobolev dimension of $\nu$ as

$$
\operatorname{dim}_{s} \nu=\sup \left\{\alpha: \int(1+|x|)^{\alpha-n}|\hat{\nu}(x)|^{2} d x<\infty\right\} .
$$

Then $0 \leq \operatorname{dim}_{s} \nu \leq \infty$.

Example. $\operatorname{dim}_{s} \delta_{0}=0$, since $\hat{\delta}_{0} \equiv 1$. It is essentially because of measures such as $\delta_{0}$, whose Fourier transform does not decay sufficiently at infinity that we used $1+|x|$ and not $|x|$ in the above definition.

The Sobolev dimension conveniently captures various properties of $\nu$ :

Proposition 5.1. Let $\nu \in \mathcal{M}\left(\mathbb{R}^{n}\right)$.

(1) If $0<\operatorname{dim}_{s} \nu \leq n$, then

$$
\operatorname{dim}_{s} \nu=\sup \left\{\alpha: I_{\alpha}(\mu)<\infty\right\} .
$$

(2) If $\operatorname{dim}_{s} \nu>n$, then $\nu \in L^{2}\left(\mathbb{R}^{n}\right)$.

(3) If $\operatorname{dim}_{s} \nu>2 n$, then $\nu$ is continuous.

Proof: (1) and (2) are clear and (3) is just a restatement of Proposition 4.4 . 
Now we describe the general setting; for more precise formulations, see $[\mathbf{P S}]$.

Let $(\Omega, d)$ be a compact metric space, let $J \subset \mathbb{R}$ be an open interval and let

$$
\pi: J \times \Omega \rightarrow \mathbb{R}
$$

be a continuous map. We define

$$
\pi_{\lambda}: \Omega \rightarrow \mathbb{R}, \quad \pi_{\lambda}(\omega)=\pi(\lambda, \omega) \text { for }(\lambda, \omega) \in J \times \Omega .
$$

We assume that for each $\ell=0,1, \ldots$ there exists a constant $C_{\ell}$ such that for $\lambda \in J$ and $\omega \in \Omega$,

$$
\left|\frac{d^{\ell}}{d \lambda^{\ell}} \pi(\lambda, \omega)\right| \leq C_{\ell} .
$$

A key property is transversality, which we here assume to hold in the following form. Set

$$
\phi_{\lambda}\left(\omega_{1}, \omega_{2}\right)=\frac{\pi\left(\lambda, \omega_{1}\right)-\pi\left(\lambda, \omega_{2}\right)}{d\left(\omega_{1}, \omega_{2}\right)}
$$

for $\lambda \in I, \omega_{1}, \omega_{2} \in \Omega$. We assume that there is a constant $C$ such that

$$
\left|\phi_{\lambda}\left(\omega_{1}, \omega_{2}\right)\right|<C \Longrightarrow\left|\frac{d}{d \lambda} \phi_{\lambda}\left(\omega_{1}, \omega_{2}\right)\right|>C .
$$

Of course it is not essential that we have the same constant on both sides.

In the following $\mu$ will be a finite Borel measure on $\Omega$. Its $\alpha$-energy is

$$
I_{\alpha}(\mu)=\iint d\left(\omega_{1}, \omega_{2}\right)^{-\alpha} d \mu \omega_{1} d \mu \omega_{2} .
$$

The goal is to find out what implications the condition $I_{\alpha}(\mu)<\infty$ has for the Sobolev dimensions of the measures $\nu_{\lambda} \in \mathcal{M}(\mathbb{R})$ defined by

$$
\nu_{\lambda}=\pi_{\lambda} \mu \text {, i.e., } \nu_{\lambda}(B)=\mu\left(\pi_{\lambda}^{-1}(B)\right) \text { for } B \subset \mathbb{R} .
$$

Example. For the orthogonal projections in $\mathbb{R}^{2}$ we can take $\Omega$ to be some closed disc in $\mathbb{R}^{2}$ and $J=(0, \pi)$. To check transversality, let $\frac{\left(\omega_{1}-\omega_{2}\right)}{\left|\omega_{1}-\omega_{2}\right|}=(u, v) \in S^{1}$. Then

$$
\begin{aligned}
\phi_{\lambda}\left(\omega_{1}, \omega_{2}\right) & =u \cos \lambda+v \sin \lambda \quad \text { and } \\
\frac{d}{d \lambda}\left(\omega_{1}, \omega_{2}\right) & =-u \sin \lambda+v \cos \lambda,
\end{aligned}
$$

from which transversality is clear. 
Roughly speaking, transversality means that if $\phi_{\lambda}\left(\omega_{1}, \omega_{2}\right)$ is very small for some $\lambda$, it does not stay very small for long when we move $\lambda$. This is good for the kind of results we are looking for: if $\mu$ is not concentrated too much on small sets (expressed by $I_{\alpha}(\mu)<\infty$ ) neither is $\nu_{\lambda}$ for a typical $\lambda$ (expressed by a lower bound on the Sobolev dimension).

We now state the main theorem. It does not only give good " $\mathcal{L}$ " almost all $\lambda$ " results but unifies and improves many earlier results on the dimensions of exceptional sets.

Theorem 5.2. Suppose $I_{\alpha}(\mu)<\infty$ for some $\alpha>0$.

(1) $\int_{J}\left\|\nu_{\lambda}\right\|_{2, \gamma}^{2} d \lambda \leq C_{\gamma} I_{\alpha}(\mu)$ if $0<1+2 \gamma \leq \alpha$.

(2) $\operatorname{dim}\left\{\lambda \in J: \operatorname{dim}_{s} \nu_{\lambda} \leq \sigma\right\} \leq 1+\sigma-\alpha$ if $0 \leq \alpha-1<\sigma \leq \alpha$.

(3) $\operatorname{dim}\left\{\lambda \in J: \operatorname{dim}_{s} \nu_{\lambda} \leq \sigma\right\} \leq \sigma$ if $0<\sigma \leq \alpha$.

Before explaining some of the key ideas behind the proof, let us see what this theorem tells us about orthogonal projections into the lines. Let $A \subset \mathbb{R}^{n}$ be a Borel set. If $\operatorname{dim} A>1$, we can take $\alpha>1, \gamma>0$ such $1+2 \gamma \leq \alpha$, and $\mu \in \mathcal{M}_{1}(A)$ such that $I_{\alpha}(\mu)<\infty$.

Then by $(1), \nu_{\lambda} \ll \mathcal{L}^{1}$ for almost all $\lambda \in(0, \pi)$, consequently $\mathcal{L}^{1}\left(p_{\lambda}(A)\right)>$ 0 , and we recover Theorem 1.1(2) in the case $m=1, n=2$. Furthermore, (2) implies that

$$
\begin{aligned}
& \operatorname{dim}\left\{\lambda \in J: \nu_{\lambda} \text { is not absolutely continuous }\right\} \\
\leq & \operatorname{dim}\left\{\lambda \in J: \operatorname{dim}_{s} \nu_{\lambda} \leq 1\right\} \leq 2-\alpha,
\end{aligned}
$$

which allows us to recover Theorem 1.2(2). Similarly, Theorem 1.2(1) follows from (3).

To apply a similar general theorem to projections $P_{V}: \mathbb{R}^{n} \rightarrow V, V \in$ $G(n, m)$, one needs to extend Theorem 5.2 to the case where $J$ is replaced by a subset of some $\mathbb{R}^{p}$ and the map $\pi$ goes into $\mathbb{R}^{m}$. Such an extension can be found in $[\mathbf{P S}]$. When $m=1$ the inequality (1) stays as it is. We can recover Theorem 4.6 from it using Proposition 5.1(3).

We now discuss a couple of key ideas in the proof of Theorem 5.2. The first is a Littlewood-Paley decomposition

$$
\|\nu\|_{2, \gamma}^{2} \approx \sum_{j=-\infty}^{\infty} 2^{2 \gamma j} \int \psi_{2^{-j}} * \nu d \nu=c \sum_{j=-\infty}^{\infty} 2^{2 \gamma j} \int \hat{\psi}_{2^{-j}}|\hat{\nu}|^{2} d \mathcal{L}^{1}
$$

where $\psi$ is a suitably chosen rapidly decaying $C^{\infty}$ function in $\mathbb{R}$ with $\hat{\psi} \geq$ 0 and $\psi_{2^{-j}}(x)=2^{j} \psi\left(2^{j} x\right)$. This is rather easy (see [PS, Lemma 4.1]) but very useful. 
It is first used to prove (1). The left hand side of (5.1) can be written as

$$
\int_{J}\left\|\nu_{\lambda}\right\|_{2, \gamma}^{2} d \lambda=\int_{\mathbb{R}}|u|^{2 \gamma} \int_{J}\left|\hat{\nu}_{\lambda}(u)\right|^{2} d \lambda d u .
$$

To estimate the $\lambda$-integral one can, assuming $J$ is bounded, put in a $C^{\infty}$ function $\rho$ with compact support such that $\rho \equiv 1$ on $J$ and reduce this to the estimation of

$$
\int_{-\infty}^{\infty}\left|\hat{\nu}_{\lambda}(u)\right|^{2} \rho(u) d u=\int_{\Omega} \int_{\Omega} \int_{-\infty}^{\infty} e^{i u d\left(\omega_{1}, \omega_{2}\right) \phi_{\lambda}\left(\omega_{1}, \omega_{2}\right)} \rho(\lambda) d \mu \omega_{1} d \mu \omega_{2},
$$

where we have just written $\left|\hat{\nu}_{\lambda}(u)\right|^{2}=\hat{\nu}_{\lambda}(u) \overline{\hat{\nu}_{\lambda}(u)}$ and used the definitions of $\nu_{\lambda}$ and $\phi_{\lambda}$. If transversality holds in a stronger sense so that $\left|\frac{d}{d \lambda} \phi_{\lambda}\left(\omega_{1}, \omega_{2}\right)\right|>C>0$ for some constant $C$ and there are good upper bounds for higher order derivatives, then this integral can be estimated by simple partial integration, see [PS, p. 212]. However, this estimate becomes much more delicate with the weaker notion of transversality that we introduced earlier. Fortunately the Littlewood-Paley decomposition helps and reduces the problem to estimation of the integrals

$$
\int_{-\infty}^{\infty} \rho(\lambda) \psi\left(2^{j} d\left(\omega_{1}, \omega_{2}\right) \phi_{\lambda}\left(\omega_{1}, \omega_{2}\right)\right) d \lambda, \quad j \in \mathbb{Z},
$$

see [PS, p. 213].

There are many technical difficulties in the proofs of both (2) and (3). Inequality (3) is proved by introducing a measure given by Frostman's lemma on the exceptional set of $\lambda$ 's. Inequality (2) requires more fundamental new ideas. The Littlewood-Paley decomposition (5.1) is also used here. To see roughly how it goes, suppose $I_{\alpha}(\mu)<\infty$ and introduce the functions $h_{j}, j \in \mathbb{Z}$, by

$$
h_{j}(\lambda)=2^{-j} \int\left(\psi_{2^{-j}} * \nu_{\lambda}\right) d \nu_{\lambda}
$$

Then $h_{j} \geq 0$ as $\hat{\psi} \geq 0$. From (5.1) we get

$$
\sum_{j=0}^{\infty} 2^{j(1+2 \gamma)} h_{j}(\lambda) \leq C\left\|\nu_{\lambda}\right\|_{2, \gamma}^{2}
$$

whence by Theorem 5.2(1)

$$
2^{j(1+2 \gamma)} \int h_{j}(\lambda) d \lambda \leq C I_{\alpha}(\mu)<\infty, \quad j=0,1, \ldots .
$$

The derivatives of $h_{j}$ are easily estimated:

$$
\left|h_{j}^{(\ell)}(\lambda)\right| \leq C_{\ell} 2^{j \ell}, \quad j, \ell=0,1 \ldots .
$$


Finally, for any $\varepsilon>0$ the definition of Sobolev dimension and (5.1) yield

$$
\left\{\lambda: \operatorname{dim}_{s}\left(\nu_{\lambda}\right) \leq \sigma\right\} \subset\left\{\lambda: \sum_{j=0}^{\infty} 2^{(\sigma+\varepsilon) j} h_{j}(\lambda)=\infty\right\} .
$$

These properties of the functions $h_{j}$ reduce the proof of Theorem 5.2(2) to the following lemma with $A=2, r=2^{\sigma+\varepsilon}$ and $R=2^{2 \gamma+1}$. Peres and Schlag have also a higher dimensional version of this interesting lemma in [PS, p. 203].

Lemma. Let $h_{j} \in C^{\infty}(J), j=0,1, \ldots$, where $J \subset \mathbb{R}$ is an open interval, and let $A>1, R>r \geq 1$. Suppose

(1) $\left|h_{j}^{(\ell)}(\lambda)\right| \leq C_{\ell} A^{j \ell}$ for $\lambda \in J, j, \ell=0,1, \ldots$, and

(2) $R^{j} \int_{J}\left|h_{j}(\lambda)\right| d \lambda \leq C$ for $j=0,1, \ldots$.

(i) If $A<\frac{R}{r}$, then

$$
\sum_{j=0}^{\infty} r^{j}\left|h_{j}(\lambda)\right|<\infty \text { for all } j \in J .
$$

(ii) If $A^{\alpha} \leq \frac{R}{r} \leq A$, then

$$
\operatorname{dim}\left\{\lambda \in J: \sum_{j=0}^{\infty} r^{j}\left|h_{j}(\lambda)\right|=\infty\right\} \leq 1-\alpha .
$$

In the proof one actually estimates the size of the larger set

where

$$
\bigcap_{k=1}^{\infty} \bigcup_{j=k}^{\infty} E_{j}
$$

$$
E_{j}=\left\{\lambda \in J:\left|h_{j}(\lambda)\right|>j^{-2} r^{j}\right\} .
$$

Each $E_{j}$ is covered by intervals of length $\approx A^{-j}$ so that the average of $\left|h_{j}\right|$ over them is at least $C j^{-2} r^{-j}$. By (2), there cannot be too many such intervals and this gives an upper bound for the Hausdorff dimension of $E_{j}$. And why can $E_{j}$ be covered with such intervals? The reason is that the bound $(1)$ on the derivatives forces $\left|h_{j}\right|$ to stay $\approx j^{-2} r^{-j}$ in an interval of length $\approx A^{-j}$ once it is at that level in one point. This is elementary but somewhat delicate; one really needs high order derivatives for it. More precisely it is deduced from the estimate

$$
\left|\sum_{i=0}^{N}\left(\begin{array}{c}
N \\
i
\end{array}\right)(-1)^{i} h_{j}(\lambda+i y)\right| \leq|y|^{N}\left\|h_{j}^{(N)}\right\|_{\infty} \leq C_{N}\left(|y| A^{j}\right)^{N},
$$

which holds for any integer $N$. 
We now discuss some applications. For many more, see the paper of Peres and Schlag. We already saw how the projection theorems can be obtained from this general theory. Now we show how the distance set result given in Theorem 3.2 can be recovered and generalized.

Pinned distance sets. For $A \subset \mathbb{R}^{n}$ and $x \in \mathbb{R}^{n}$ let

$$
D_{x}(A)=\{|x-y|: y \in A\}
$$

be the "pinned" distance set of $A$ from $x$. We assume $A$ is bounded and choose $\Omega$ to be some closed ball in $\mathbb{R}^{n}$ containing $A$. We take $J$ to be $\mathbb{R}^{n}$, so we need the version where $J$ is more than one-dimensional. The maps $\pi_{\lambda}, \lambda \in \mathbb{R}^{n}$, are given by

$$
\pi_{\lambda}(\omega)=|\omega-\lambda|^{2}
$$

we use the square just to have more smoothness. The concept of transversality is technically more complicated in the higher dimensional case and we don't formulate it here. However, the main content is the same: when $\left|\phi_{\lambda}\left(\omega_{1}, \omega_{2}\right)\right|$ is small the derivative $\left|D_{\lambda} \phi_{\lambda}\left(\omega_{1}, \omega_{2}\right)\right|$ must not be too small. In our case $\left|D_{\lambda} \phi_{\lambda}\left(\omega_{1}, \omega_{2}\right)\right|=2$ and transversality trivially holds.

Suppose now $\operatorname{dim} A>\frac{(n+1)}{2}$ and choose $\frac{(n+1)}{2}<\alpha<\operatorname{dim} A$ and $\mu \in \mathcal{M}(A)$ such that $I_{\alpha}(\mu)<\infty$. Then the higher dimensional version of Theorem 5.2(2) yields

$$
\begin{aligned}
& \operatorname{dim}\left\{\lambda: \mathcal{L}^{1}\left(D_{\lambda}(A)\right)=0\right\} \\
\leq & \operatorname{dim}\left\{\lambda: \nu_{\lambda} \perp \mathcal{L}^{1}\right\} \\
\leq & \operatorname{dim}\left\{\lambda: \operatorname{dim}_{s} \nu_{\lambda} \leq 1\right\} \leq n+1-\alpha .
\end{aligned}
$$

Since this holds for all $\alpha<\operatorname{dim} A$, we have

$$
\operatorname{dim}\left\{\lambda: \mathcal{L}^{1}\left(D_{\lambda}(A)\right)=0\right\} \leq n+1-\operatorname{dim} A<\frac{(n+1)}{2} .
$$

Since the right hand side is less than $\operatorname{dim} A$ and $D_{\lambda}(A) \subset D(A)$ for $\lambda \in A$, this generalizes Theorem 3.2.

In a similar way one gets that if $\operatorname{dim} A>\frac{(n+2)}{2}$, then

$$
\operatorname{dim}\left\{\lambda: \operatorname{Int} D_{\lambda}(A)=\emptyset\right\} \leq n+2-\operatorname{dim} A<\frac{(n+2)}{2} .
$$

Recall that for $D(A)$ we know more: $\operatorname{dim} A>\frac{(n+1)}{2} \operatorname{implies} \operatorname{Int} D(A) \neq$ $\emptyset$.

It may be that for pinned distance sets the correct lower bound for $\operatorname{dim} A$ is also $\frac{n}{2}$. 
Bernoulli convolutions. This is not a geometric problem but the generalized projections also work well in this case. For much more on this topic, see $[\mathbf{P S}]$ and the references given there, in particular, the nice survey article $[\mathbf{P S S}]$. For $0<\lambda<1$, let $\nu_{\lambda}$ be the distribution of the random series $\sum_{i=0}^{\infty} \pm \lambda^{i}$ where the signs + and - are chosen with equal probability. This is put into the general scheme in the following way. We let

$$
\Omega=\{-1,1\}^{\mathbb{N}}=\left\{\left(\omega_{i}\right): \omega_{i}=-1 \text { or } \omega_{i}=1 \text { for } i=0,1, \ldots\right\} .
$$

The compact product topology of $\Omega$ can be metrized in many ways. Here a convenient metric $d$ is defined by choosing $\lambda_{1} \in(0,1)$ and letting

$$
d\left(\left(\omega_{i}\right),\left(\tau_{i}\right)\right)=\lambda_{1}^{-i_{0}} \text { with } i_{0}=\min \left\{i: \omega_{i} \neq \tau_{i}\right\} .
$$

Let $\mu$ be the countable product of the measures $\frac{1}{2}\left(\delta_{\{1\}}+\delta_{\{-1\}}\right)$. Then $\mu$ is determined by its values on the cylinder sets:

$$
\mu\left(\left\{\left(\omega_{i}\right): \omega_{i}=\tau_{i} \text { for } 0 \leq i<n\right\}\right)=2^{-n} \text { for }\left(\tau_{0}, \ldots, \tau_{n-1}\right) \in\{-1,1\}^{n} .
$$

It is easy to check that $I_{\alpha}(\mu)<\infty$ if and only if $\lambda_{1}^{\alpha}>\frac{1}{2}$. The maps $\pi_{\lambda}$ are defined by

$$
\pi_{\lambda}\left(\left(\omega_{i}\right)\right)=\sum_{i=0}^{\infty} \omega_{i} \lambda^{i}
$$

Then for $\nu_{\lambda}=\pi_{\lambda} \omega$,

$$
\nu_{\lambda}(A)=\mu\left(\left\{\left(\omega_{i}\right): \sum_{i=0}^{\infty} \omega_{i} \lambda^{i} \in A\right\}\right) \text { for } A \subset \mathbb{R} .
$$

It was a central open problem for a long time whether or not $\nu_{\lambda}$ is absolutely continuous for almost all $\lambda \in\left(\frac{1}{2}, 1\right)$. It is easy to see that it is singular for $\lambda \in\left(0, \frac{1}{2}\right)$. It is more difficult but true that $\nu_{\lambda}$ is singular for $\lambda$ in a countable dense subset of $\left(\frac{1}{2}, 1\right)$. Solomyak solved this problem in 1995 in $[\mathbf{S}]$ by proving that $\nu_{\lambda} \ll \mathcal{L}^{1}$ for almost all $\lambda \in\left(\frac{1}{2}, 1\right)$. The general projection theorems of Peres and Schlag give this in a sharper form: there is a constant $C$ such that for all small $\varepsilon>0$

$$
\operatorname{dim}\left\{\lambda \in\left(\frac{1}{2}+\varepsilon, 1\right): \nu_{\lambda} \text { is singular }\right\} \leq 1-C \varepsilon .
$$

This may be far from sharp since it is not known if the exceptional set is countable. 


\section{Visibility problems}

For simplicity, we shall mainly discuss these in the plane although in most cases there are higher dimensional versions. Let $A \subset \mathbb{R}^{2}$ be a Borel set. We shall say that $A$ is

$$
\begin{array}{ll}
\text { invisible, } & \text { if } \mathcal{L}^{1}\left(p_{\theta}(A)\right)=0 \text { for almost } \theta \in[0, \pi), \\
\text { visible, } & \text { if } \mathcal{L}^{1}\left(p_{\theta}(A)\right)>0 \text { for almost all } \theta \in[0, \pi), \\
\text { partially visible, } & \text { if } \mathcal{L}^{1}\left(\left\{\theta \in(0, \pi): \mathcal{L}^{1}\left(p_{\theta}(A)\right)>0\right\}\right)>0 .
\end{array}
$$

We shall also study visibility from a point. Let $a \in \mathbb{R}^{2}$ and let $\pi_{a}$ be the radial projection from a:

$$
\pi_{a}: \mathbb{R}^{2} \backslash\{a\} \longrightarrow S^{1}, \quad \pi_{a}(x)=\frac{(x-a)}{|x-a|} .
$$

We shall say that $A$ is

invisible from $A$, if $\mathcal{H}^{1}\left(\pi_{a}(A \backslash\{a\})\right)=0$,

visible from $A, \quad$ if $\mathcal{H}^{1}\left(\pi_{a}(A \backslash\{a\})\right)>0$.

Clearly, if $\mathcal{H}^{1}(A)=0$, then $A$ is invisible and it is invisible from all points. If $\operatorname{dim} A>1$ (or only $C_{1}(A)>0$ ), $A$ is visible by Theorem 1.1(2). It is also visible from $\mathcal{L}^{2}$ almost all points $a \in \mathbb{R}^{2}$, as can be seen by a simple modification of the proof of Theorem 1.1(2).

An application of the Peres-Schlag machinery gives more precisely

$$
\operatorname{dim}\left\{a \in \mathbb{R}^{2}: A \text { is invisible from } A\right\} \leq 2-\operatorname{dim} A .
$$

If $0<\mathcal{H}^{1}(A)<\infty$, Besicovitch's projection theorem tells us that $A$ is invisible if and only if $\mathcal{H}^{1}(A \cap \Gamma)=0$ for every rectifiable curve $\Gamma$, that is, $A$ is purely unrectifiable. Otherwise it is obviously visible.

But from how big a set $B$ can such a purely unrectifiable set $A$ with $\mathcal{H}^{1}(A)<\infty$ be visible? It is rather easy to see that $\mathcal{L}^{2}(B)=0$. Marstrand proved in $[\mathbf{M}]$ that $\operatorname{dim} B \leq 1$. In $[\mathbf{M} 1]$ it was shown that for any invisible Borel set $A$ (which could have $\mathcal{H}^{1}(A)=\infty$ ) the corresponding set $B$ is purely unrectifiable in the sense that $\mathcal{H}^{1}(B \cap \Gamma)=0$ for every rectifiable curve $\Gamma$ and the capacity $C_{1}(B)=0$. A generalization of both of these statements would be that $B$ is also invisible. Unfortunately this is false as shown by Csörnyei in $[\mathbf{C 1}]$. In $[\mathbf{C 2}]$, she gave a characterization of such sets $B$.

There is an interesting and probably quite difficult question related to the pointwise visibility of a purely unrectifiable Borel set $A \subset \mathbb{R}^{2}$ with $\mathcal{H}^{1}(A)<\infty$ : is $A$ invisible from $\mathcal{H}^{1}$ almost all points $a \in A$ ? A closely related question for any Borel set $A \subset \mathbb{R}^{2}$ with $\mathcal{H}^{1}(A)<\infty$ is: is it true 
that for $\mathcal{H}^{1}$ almost all $x \in A$ the set $A \cap L$ is finite for almost all lines through $x$ ?

Strange things can occur with the visibility of Borel sets $A$ with $\mathcal{H}^{1}(A)=\infty$ and $\operatorname{dim} A=1$. One might think that partially visible Borel sets are always visible, but this is not so. Talagrand showed in $[\mathbf{T}]$ that if $f:[0, \pi) \rightarrow[0, \infty)$ is any upper semicontinuous function, then there is a compact set $C \subset \mathbb{R}^{2}$ such that

$$
\mathcal{L}^{1}\left(p_{\theta}(C)\right)=f(\theta) \text { for } \theta \in[0, \pi) .
$$

Falconer observed in [F2, Theorem 7.11] that as a consequence of a result of Davies, one can not only give in advance the measures of the projections but also the projections as sets in the following sense.

Theorem 6.1. Let $A_{\theta} \subset \mathbb{R}$ for $\theta \in[0, \pi)$ be such that $\left\{(\theta, y): y \in A_{\theta}\right\}$ is $\mathcal{L}^{2}$ measurable. Then there exists a Borel set $A \subset \mathbb{R}^{2}$ such that for almost all $\theta \in[0, \pi)$

$$
A_{\theta} \subset p_{\theta}(A) \text { and } \mathcal{L}^{1}\left(p\left(A_{\theta}\right) \backslash A_{\theta}\right)=0 .
$$

This is obtained by duality principles between lines and points similar to those used in Section 4. Falconer proved a higher dimensional version of this in $[\mathbf{F 4}]$, see also [F5]. Then a concrete construction is needed.

What happens to visibility under smooth maps? If $\mathcal{H}^{1}(A)<\infty$ with $A$ a Borel set in $\mathbb{R}^{2}, A$ is invisible if and only if it is purely unrectifiable, otherwise it is visible. It is clear that bilipschitz maps preserve pure unrectifiability, whence they also preserve the visibility and invisibility in the case $\mathcal{H}^{1}(A)<\infty$. Similarly they preserve Hausdorff dimension and hence the visibility of Borel sets $A$ with $\operatorname{dim} A>1$. But the case when $\mathcal{H}^{1}(A)=\infty$ and $\operatorname{dim} A=1$ is again different. It was shown in [M3] that the only $C^{2}$ diffeomorphisms $f: \mathbb{R}^{2} \rightarrow \mathbb{R}^{2}$ that preserve all invisible compact sets are the affine ones. This was used to disprove a conjecture of Vitushkin. He asked whether a compact subset of $\mathbb{C}$ could be removable for bounded analytic functions if and only if it is invisible. (For removability see, e.g., [M6, Chapter 19] or $[\mathbf{P}]$.) The answer is no since removability is trivially preserved under conformal maps. However, this did not say which of the two possible implications is false, or whether they are both false. In [JM ] Jones and Murai constructed a non-removable invisible set. Another construction of such a set was given by Joyce and Mrters in [JMö]. It is still an open question whether all removable sets are invisible. With new tools and the results of Mel'nikov in $[\mathbf{M e}]$, this question can be asked without any reference to analytic functions: 
Let $c(x, y, z)$ be the Menger curvature of the triple $x, y, z \in \mathbb{R}^{2}$. That is, $c(x, y, z)=\frac{1}{R}$ where $R$ is the radius of the circle passing through $x$, $y$ and $z$. Let $C \subset \mathbb{R}^{2}=\mathbb{C}$ be compact. Mel'nikov proved that $C$ is non-removable if there exists $\mu \in \mathcal{M}(C)$ such that

$$
\begin{gathered}
\mu(B(z, r)) \leq r \text { for } z \in \mathbb{R}^{2}, r>0, \quad \text { and } \\
\iiint c(x, y, z)^{2} d \mu x d \mu y d \mu z<\infty .
\end{gathered}
$$

Later Tolsa showed in [To] that this is also necessary for the nonremovability of $F$. Thus the question of whether all removable sets are invisible can be stated as:

Is it true that if $C \subset \mathbb{R}^{2}$ is a partially visible compact set, then there is $\mu \in \mathcal{M}(F)$ such that (6.2) and (6.3) hold?

We briefly discuss visible parts of sets from lines. Let $C \subset \mathbb{R}^{2}$ be compact and let $L \subset \mathbb{R}^{2}$ be a line such that $F \cap L=\emptyset$, and let $P_{L}: \mathbb{R}^{2} \rightarrow$ $L$ be the orthogonal projection onto $L$. We define the visible part of $C$ from $L, V_{L}(C)$, to be the set of points $x \in C$ such that the open line segment $\left(x, P_{L}(x)\right)$ does not meet $C$. This means that $V_{L}(C)$ consists of the points where we first hit $C$ leaving $L$ orthogonally. The following question is open:

Is it true that for every compact set $C \subset \mathbb{R}^{2}, \operatorname{dim} V_{L}(C) \leq 1$ for almost all lines $L$ with $C \cap L=\emptyset$ ?

Partial results have been obtained by E. and M. Järvenpää, MacManus and O'Neil in [JJMO] and by O'Neil in [O3]. In particular, they showed using the Peres-Schlag results of Section 5 that if $\operatorname{dim} C \leq 1$, then $\operatorname{dim} V_{L}(C)=\operatorname{dim} C$ for almost all lines $L$ with $C \cap L=\emptyset$. We can easily reduce the question to the lines $L$ such that $C$ lies in one half-plane whose boundary is $L$. Then a projection-type mapping $\pi_{C, L}$ such that $\pi_{C, L}(C)=V_{L}(C)$ is obtained by letting $\pi_{C, L}(x)$ be the first orthogonal hit of $C$ from $L$ as described above.

We end this survey by briefly mentioning some other topics related to projections. A local version of the Besicovitch-Federer projection theorem was given in $[\mathbf{O} 1]$. Multifractal spectra of projected measures have benn examined in $[\mathbf{O}]$ and $[\mathbf{O 2}]$. Box counting and packing dimensions behave very differently from Hausdorff dimension under orthogonal projections. They have been studied in $[\mathbf{J 1}],[\mathbf{F H 1}],[\mathbf{F H 2}],[\mathbf{H}],[\mathbf{F M}],[\mathbf{F O}]$ and $[\mathbf{O 2}]$. Their measurability properties were investigated in $[\mathbf{M M}]$. Their behaviour under intersections with planes and with more general sets were studied for example in $[\mathbf{F M}],[\mathbf{F J}],[\mathbf{J} 2],[\mathbf{C} 3]$ and $[\mathbf{J J L}]$. In $[\mathbf{Z}]$, Zähle introduced the average dimension of measures and showed that it 
behaves under projections as Hausdorff dimension. Llorente studied its intersection properties in $[\mathbf{L}]$.

Sauer and Yorke in $[\mathbf{S Y}]$, and Hunt and Kaloshin in [HK1] and [HK2 investigated transformation of dimensions under typical smooth mappings, also in infinite dimensional spaces. Ledrappier and Lindenstrauss proved in $[\mathbf{L L}]$ a result analogous to Theorem 1.1 for measures on Riemann surfaces that are invariant under geodesic flow. The interesting feature in this is that they looked only at one natural projection from the tangent bundle but were able to use the methods that we have discussed earlier for the case of typical projections.

\section{Open problems}

Here we collect many open problems that were mentioned in the text. We also state some others that we did not mention earlier.

Problem 1. For which values of $d, 0<d \leq \frac{1}{4}$, is $\mathcal{H}^{s_{d}}\left(p_{\theta}\left(C_{d}\right)\right)>0$ for almost all $\theta \in[0, \pi)$, where $C_{d}$ is the Cantor set of (1.5)? Recall that this holds if $d<\frac{1}{9}$, it does not hold if $d>\frac{1}{6}$, and the case $\frac{1}{9} \leq d \leq \frac{1}{6}$ is open.

Problem 2. Let $K$ be a compact self-similar subset of $\mathbb{R}^{2}$. That is, $K=\bigcup_{i=1}^{m} S_{i}(K)$ where each $S_{i}$ is a contractive similarity. For $C_{d}, S_{i}$ is of the form $S_{i}(x)=\frac{1}{4} x+a_{i}$. But what can be said about the measures $\mathcal{H}^{s}\left(p_{\theta}(K)\right)$ if $s=\operatorname{dim} K<1$ and the $S_{i}$ 's contain also rotations?

Problem 3. What is the best decay for

$$
\int_{0}^{\pi} \mathcal{L}^{1}\left(p_{\theta}\left(U_{k}^{\frac{1}{4}}\right)\right) d \theta \text { as } k \longrightarrow \infty \text { ? }
$$

Recall (2.1).

Problem 4. Find a new proof for Besicovitch's projection theorem for purely unrectifiable sets with $\mathcal{H}^{1}(A)<\infty$ that would give some quantitative estimates, for example for $\int_{0}^{\pi} \mathcal{L}^{1}\left(p_{\theta}(A(\varepsilon))\right) d \theta$. This could lead to new characterizations of the uniformly rectifiable sets of David and Semmes (see $[\mathbf{D S}]$ ). White has given in $[\mathbf{W}]$, a new proof for Federer's higher dimensional generalization, but it is an induction argument and relies upon Besicovitch's theorem in $\mathbb{R}^{2}$.

Problem 5. Is there $b(n, m)$ such that

$$
\int \mathcal{L}^{m}\left(P_{V}(A)\right) \geq b(n, m) \mathcal{L}^{n}(A)^{\frac{m}{n}}
$$


for all Borel sets $A \subset \mathbb{R}^{n}$ with equality for balls? Recall from (2.3) that this is true for $m=n-2 \geq 1$ and for $m=1$. Recall also the related problem for capacities from (2.2). As far as I know, the following isoperimetric problem for capacities is also open when $s \neq n-2$ : is it true that

$$
C_{s}(A) \geq C_{s}(B(0,1))
$$

for all Borel sets $A \subset \mathbb{R}^{n}$ with $\mathcal{L}^{n}(A)=\mathcal{L}^{n}(B(0,1))$ ? In fact, for $s=n-1$ this would give the sharp form of (2.3) since the sharp inequality in $(2.2)$ is then known.

Problem 6. For which gauge functions $h$ does $\Lambda_{h}(A)>0$ imply $\mathcal{L}^{1}\left(p_{\theta}(A)\right)>0$ for almost all $\theta \in[0, \pi)$ and for Borel sets $A \subset \mathbb{R}^{2}$ ? See [M6] for the definition of the Hausdorff $h$-measure $\Lambda_{h}$. If $\int_{0}^{1} \frac{h(r)}{r^{2}} d r<$ $\infty$, then $\Lambda_{h}(A)>0$ implies $C_{1}(A)>0$ and so this is true. This is rather sharp: Joyce and Mrters showed in [JM̈̈] that there exist an $h$ and a compact $C \subset \mathbb{R}^{2}$ such that $\int_{0}^{1} \frac{h(r)^{\alpha}}{r^{\alpha+1}} d r<\infty$ for all $\alpha>1, \Lambda_{h}(C)>0$ and $\mathcal{L}^{1}\left(p_{\theta}(C)\right)=0$ for all $\theta \in[0, \pi)$.

After the first version of this paper, Yuval Peres informed me that he can essentially solve this problem. Given a monotone regularly varying gauge function $h$ with $\int_{0}^{1} \frac{h(r)}{r^{2}} d r=\infty$, he can construct a random compact set $A \subset \mathbb{R}^{2}$ with $\Lambda_{h}(A)>0$ such that almost surely $\mathcal{L}^{1}\left(p_{\theta}(A)\right)=0$ for almost all $\theta \in[0, \pi)$. The argument follows the random construction Peres and Solomyak used in [PSo].

Problem 7. Modify the construction of the Cantor set $C_{\frac{1}{4}}$ in (1.5) by replacing the ratio $\frac{1}{4}$ by $\lambda_{k}$ at the step $k$. Denote this Cantor set by $C(\lambda)$ where $\lambda$ is the sequence $\left(\lambda_{k}\right)$. For which sequences $\lambda$ is $\mathcal{L}^{1}\left(p_{\theta}(C(\lambda))\right)=0$ for almost all $\theta \in[0, \pi)$ ?

Note that $\mathcal{H}^{1}(C(\lambda))<\infty$ if and only if $\sup _{n} 4^{n} \sigma_{n}<\infty$ where $\sigma_{n}=\lambda_{1} \ldots \lambda_{n}$ is the side-length of the $n$th generation squares. Let $h:[0, \infty) \rightarrow[0, \infty)$ be an increasing function such that $h\left(\sigma_{n}\right)=4^{-n}$. Then $0<\Lambda_{h}(C(\lambda))<\infty$. Hence by the remarks in the previous problem, $\mathcal{L}^{1}\left(p_{\theta}(C(\lambda))\right)>0$ for almost all $\theta \in[0, \pi)$ if $\int_{0}^{1} \frac{h(r)}{r^{2}} d r<\infty$. In terms of the sequence $\left(\lambda_{k}\right)$, this means $\sum_{n} 4^{-n} \sigma_{n}^{-1}<\infty$.

It follows from Proposition 7.2 in [PSo] that $4^{n} \sigma_{n}$ can tend to infinity at some explicit, but very slow, rate and we still have $\mathcal{L}^{1}\left(p_{\theta}(C(\lambda))\right)=0$ for almost all $\theta \in[0, \pi)$.

Problem 8. What is the smallest number $c(n, m), n \geq 3$, such that $\operatorname{dim} A>c(n, m)$ implies Int $P_{V}(A) \neq \emptyset$ for $\gamma_{n, m}$ almost all $V \in G(n, m)$ ? 
We know from Theorem 4.6 and the discussion there that $c(n, 1)=2$ and $c(n, m) \leq 2 m$.

Problem 9. The integralgeometric measure $I_{t}^{m}, 1 \leq t \leq \infty$, is constructed by Carathéodory's construction from

$$
\left\|\mathcal{L}^{m}\left(P_{V}(E)\right)\right\|_{L^{t}\left(G(n, m), \gamma_{n, m}\right)},
$$

see [Fe, 2.10.5]. It was shown in [M2] that $I_{1}^{1} \neq I_{t}^{1}$ for $t>1$, but is it also the case that $J_{t_{1}}^{1} \neq J_{t_{2}}^{1}$ for $1<t_{1}<t_{2}$ ?

Problem 10. Are all removable sets for bounded analytic functions invisible? Recall from Section 6 in particular the formulation in terms of measures satisfying (6.2) and (6.3).

The Cantor sets $C(\lambda)$ of Problem 7 are removable if and only if $\sum_{n} 4^{-2 n} \sigma_{n}^{-2}=\infty$, see [P, Section 4.3].

Problem 11. Is $\operatorname{dim} V_{L}(C) \leq 1$ for almost all lines $L$ with $C \cap L=\emptyset$ ? The visible part $V_{L}(C)$ of the compact set $C$ was defined in Section 6 .

Problem 12. Let $A$ be a Borel set in $\mathbb{R}^{2}$ with $\mathcal{H}^{1}(A)<\infty$. Is it true that for $\mathcal{H}^{1}$ almost all $x \in A$, the intersection $A \cap L$ is a finite set for almost all lines $L$ through $x$ ? If $A$ is purely unrectifiable, one can ask further whether these intersections contain only $x$. This problem was mentioned in Section 6.

Problem 13. Is $\operatorname{dim} B=n$ for every set $B \subset \mathbb{R}^{n}$ which contains a line in every direction? By Theorem 4.2 this is true for $n=2$.

Problem 14. For which integers $0<m<n$ do there exist Borel sets $B \subset \mathbb{R}^{n}$ with $\mathcal{L}^{n}(B)=0$ that contain an $m$-plane in every direction? Some partial results were discussed in Section 4.

Problem 15. What is the smallest number $c(n), n \geq 2$, such that $\operatorname{dim} A>c(n)$ implies $\mathcal{L}^{1}(D(A))>0$ for all Borel sets $A \subset \mathbb{R}^{n}$ ? By Theorems 3.2 and 3.3 we know that $c(n) \leq \frac{(n+1)}{2}$ and $c(2) \leq \frac{4}{3}$. Also $c(n) \geq \frac{n}{2}$ for all $n \geq 2$.

Problem 16. What is the smallest number $b(n), n \geq 2$, such that $\operatorname{dim} A>b(n)$ implies $\operatorname{Int} D(A) \neq \emptyset$ for all Borel sets $A \subset \mathbb{R}^{n}$ ? By Theorem 3.4, $b(n) \leq \frac{(n+1)}{2}$, and again $b(n) \geq \frac{n}{2}$.

Problem 17. Questions analogous to Problems 14 and 15 for the pinned distance sets that were discussed in Section 5 .

Problem 18. Let $A, B \subset \mathbb{R}^{n}$ be Borel sets. Is $\mathcal{L}^{n}\left(\left\{z \in \mathbb{R}^{n}: \operatorname{dim}(A \cap\right.\right.$ $(g(B)+z)) \geq \operatorname{dim} A+\operatorname{dim} B-n\})>0$ for almost all rotations $g \in O(n)$ ? 
This is true if $\operatorname{dim} A \geq \frac{(n+1)}{2}$ or $\operatorname{dim} B \geq \frac{(n+1)}{2}$, see [M6, Theorem 13.11]. The proof uses the Fourier transform and $\frac{(n+1)}{2}$ comes from similar reasons as for the distance sets.

Problem 19. What is $\alpha(s)$ in $\mathbb{R}^{n}$ ? Recall the discussion on partial results in Section 3 and Wolff's solution in $\mathbb{R}^{2}$ in Theorem 3.1.

Problem 20. For which values of $s, 0<s<n$, does there exist $\mu \in$ $\mathcal{M}\left(\mathbb{R}^{n}\right)$ such that

$$
\frac{r^{s}}{C} \leq \mu(B(x, r)) \leq C r^{s} \quad \text { for } x \in \operatorname{spt} \mu, \quad 0<r<1,
$$

and

$$
|\hat{\mu}(x)| \leq|x|^{-\frac{s}{2}} \quad \text { for } x \in \mathbb{R}^{n} ?
$$

As mentioned in Section 3, this was asked by Mitsis in [Mi2], and the answer could be: only for the integers $s$.

\section{References}

[BL] A. BAERnstein, II AND M. Loss, Some conjectures about $L^{p}$ norms of $k$-plane transforms, Rend. Sem. Mat. Fis. Milano 67 (1997), 9-26 (2000).

[BG] C. BAndt And S. Graf, Self-similar sets. VII. A characterization of self-similar fractals with positive Hausdorff measure, Proc. Amer. Math. Soc. 114(4) (1992), 995-1001.

[B] C. Bluhm, On a theorem of Kaufman: Cantor-type construction of linear fractal Salem sets, Ark. Mat. 36(2) (1998), 307-316.

[B1] J. Bourgain, Besicovitch type maximal operators and applications to Fourier analysis, Geom. Funct. Anal. 1(2) (1991), 147-187.

[B2] J. Bourgain, Hausdorff dimension and distance sets, Israel J. Math. 87(1-3) (1994), 193-201.

[C1] M. Csrnyei, On the visibility of invisible sets, Ann. Acad. Sci. Fenn. Math. 25(2) (2000), 417-421.

[C2] M. CsRnyei, How to make Davies' theorem visible, Bull. London Math. Soc. 33(1) (2001), 59-66.

[C3] M. CsRnyeI, On planar sets with prescribed packing dimensions of line sections, Math. Proc. Cambridge Philos. Soc. 130(3) (2001), 523-539. 
[DS] G. DAVID AND S. SEmmes, "Analysis of and on uniformly rectifiable sets", Mathematical Surveys and Monographs 38, American Mathematical Society, Providence, RI, 1993.

[DM] J. J. DiJkstra AND J. VAn Mill, Projections of planar Cantor sets in potential theory, Indag. Math. (N.S.) 8(2) (1997), 173-180.

[EM] G. A. Edgar And C. Miller, Borel subrings of the reals, Proc. Amer. Math. Soc. 131(4) (2003), 1121-1129 (electronic).

[F1] K. J. FALCONER, Hausdorff dimension and the exceptional set of projections, Mathematika 29(1) (1982), 109-115.

[F2] K. J. FALCONER, "The geometry of fractal sets", Cambridge Tracts in Mathematics 85, Cambridge University Press, Cambridge, 1986.

[F3] K. J. FALCONER, On the Hausdorff dimensions of distance sets, Mathematika 32(2) (1985), 206-212 (1986).

[F4] K. J. FALCONER, Sets with prescribed projections and Nikodým sets, Proc. London Math. Soc. (3) 53(1) (1986), 48-64.

[F5] K. J. FAlConer, Digital sundials, paradoxical sets, and Vitushkin's conjecture, Math. Intelligencer 9(1) (1987), 24-27.

[FH1] K. J. FAlCONER AND J. D. Howroyd, Projection theorems for box and packing dimensions, Math. Proc. Cambridge Philos. Soc. 119(2) (1996), 287-295.

[FH2] K. J. FAlConer And J. D. Howroyd, Packing dimensions of projections and dimension profiles, Math. Proc. Cambridge Philos. Soc. 121(2) (1997), 269-286.

[FJ] K. J. FAlCONER AND M. JÄrvenpä̈̈, Packing dimensions of sections of sets, Math. Proc. Cambridge Philos. Soc. 125(1) (1999), 89-104.

[FM] K. J. FAlCONER AND P. Mattila, The packing dimension of projections and sections of measures, Math. Proc. Cambridge Philos. Soc. 119(4) (1996), 695-713.

[FO] K. J. FAlconer And T. C. O'NeIL, Convolutions and the geometry of multifractal measures, Math. Nachr. 204 (1999), 61-82.

[Fe] H. FEDERER, "Geometric measure theory", Die Grundlehren der mathematischen Wissenschaften 153, Springer-Verlag New York Inc., New York, 1969.

[G] M. DE GuzMán, "Real variable methods in Fourier analysis", North-Holland Mathematics Studies 46, Notas de Matemática 75, North-Holland Publishing Co., AmsterdamNew York, 1981. 
[H] J. D. Howroyd, Box and packing dimensions of projections and dimension profiles, Math. Proc. Cambridge Philos. Soc. 130(1) (2001), 135-160.

[HK1] B. R. Hunt AND V. Yu. Kaloshin, How projections affect the dimension spectrum of fractal measures, Nonlinearity 10(5) (1997), 1031-1046.

[HK2] B. R. Hunt And V. Yu. Kaloshin, Regularity of embeddings of infinite-dimensional fractal sets into finite-dimensional spaces, Nonlinearity 12(5) (1999), 1263-1275.

[JJ1] E. JäRVENP $\ddot{A} \ddot{A}$ AND M. JÄRVENPÄ̈̈, Linear mappings and generalized upper spectrum for dimensions, Nonlinearity 12(3) (1999), 475-493.

[JJ2] E. JÄRVENPÄ̈̈ AND M. JÄRVENPÄÄ, On the definition of SRB-measures for coupled map lattices, Comm. Math. Phys. 220(1) (2001), 1-12.

[JJL] E. JÄrvenpä̈̈, M. JÄrvenpä̈̈ And M. Llorente, Local dimensions of sliced measures and stability of packing dimensions of sections of sets, Adv. Math. (to appear).

[JJMO] E. J̈̈RVEnPÄ̈̈, M. Järvenpä̈̈, P. MacManus And T. C. O'NeIL, Visible parts and dimensions, Nonlinearity 16(3) (2003), 803-818.

[J1] M. J $\ddot{A} R V E N P \ddot{A} \ddot{A}$, On the upper Minkowski dimension, the packing dimension, and orthogonal projections, Ann. Acad. Sci. Fenn. Ser. A I Math. Dissertationes 99 (1994), 34 pp.

[J2] M. JÄRVEnPÄ̈̈, Hausdorff and packing dimensions, intersection measures, and similarities, Ann. Acad. Sci. Fenn. Math. 24(1) (1999), 165-186.

[JKV] P. W. Jones, N. H. Katz And A. VArgas, Checkerboards, Lipschitz functions and uniform rectifiability, Rev. Mat. Iberoamericana 13(1) (1997), 189-210.

[JM] P. W. Jones AND T. Murai, Positive analytic capacity but zero Buffon needle probability, Pacific J. Math. 133(1) (1988), 99-114.

[JMö] H. Joyce AND P. MÖrTers, A set with finite curvature and projections of zero length, J. Math. Anal. Appl. 247(1) (2000), 126-135.

[K] J.-P. Kahane, "Some random series of functions", Second edition, Cambridge Studies in Advanced Mathematics 5, Cambridge University Press, Cambridge, 1985. 
[KS] J.-P. Kahane AND R. SAlEm, "Ensembles parfaits et séries trigonométriques", Actualités Sci. Indust. 1301, Hermann, Paris, 1963.

[KT] N. H. Katz And T. TAO, Some connections between Falconer's distance set conjecture and sets of Furstenberg type, New York J. Math. 7 (2001), 149-187 (electronic).

[K1] R. Kaufman, On Hausdorff dimension of projections, Mathematika 15 (1968), 153-155.

[K2] R. Kaufman, On the theorem of Jarník and Besicovitch, Acta Arith. 39(3) (1981), 265-267.

[KM] R. Kaufman And P. Mattila, Hausdorff dimension and exceptional sets of linear transformations, Ann. Acad. Sci. Fenn. Ser. A I Math. 1(2) (1975), 387-392.

[Ke] R. KenYon, Projecting the one-dimensional Sierpinski gasket, Israel J. Math. 97 (1997), 221-238.

[LT] I. LABA AND T. TAO, An improved bound for the Minkowski dimension of Besicovitch sets in medium dimension, Geom. Funct. Anal. 11(4) (2001), 773-806.

[LL] F. Ledrappier AND E. Lindenstrauss, On the projections of measures invariant under the geodesic flow, Int. Math. Res. Not. 9 (2003), 511-526.

[L] M. Llorente, On the behaviour of the average dimension: sections, products and intersection measures, Dissertation, University of Jyväskylä, Helsinki, 2002, Ann. Acad. Sci. Fenn. Math. Diss. 126 (2002), 47 pp.

[M] J. M. MARstrand, Some fundamental geometrical properties of plane sets of fractional dimensions, Proc. London Math. Soc. (3) 4 (1954), 257-302.

[M1] P. Mattila, Integralgeometric properties of capacities, Trans. Amer. Math. Soc. 266(2) (1981), 539-554.

[M2] P. Mattila, An example illustrating integralgeometric measures, Amer. J. Math. 108(3) (1986), 693-702.

[M3] P. MatTila, Smooth maps, null-sets for integralgeometric measure and analytic capacity, Ann. of Math. (2) 123(2) (1986), 303-309.

[M4] P. MATtiLA, Spherical averages of Fourier transforms of measures with finite energy; dimension of intersections and distance sets, Mathematika 34(2) (1987), 207-228.

[M5] P. Mattila, Orthogonal projections, Riesz capacities, and Minkowski content, Indiana Univ. Math. J. 39(1) (1990), 185-198. 
[M6] P. Mattila, "Geometry of sets and measures in Euclidean spaces. Fractals and rectifiability", Cambridge Studies in Advanced Mathematics 44, Cambridge University Press, Cambridge, 1995.

[MM] P. Mattila and R. D. Mauldin, Measure and dimension functions: measurability and densities, Math. Proc. Cambridge Philos. Soc. 121(1) (1997), 81-100.

[MS] P. Mattila AND P. SJÖLin, Regularity of distance measures and sets, Math. Nachr. 204 (1999), 157-162.

[Me] M. S. MEL'NikOv, Analytic capacity: a discrete approach and the curvature of measure, (Russian), Mat. Sb. 186(6) (1995), 57-76; translation in: Sb. Math. 186(6) (1995), 827-846.

[Mi1] T. Mitsis, A note on the distance set problem in the plane, Proc. Amer. Math. Soc. 130(6) (2002), 1669-1672 (electronic).

[Mi2] T. Mitsis, A Stein-Tomas restriction theorem for general measures, Publ. Math. Debrecen 60(1-2) (2002), 89-99.

[Mi3] T. Mitsis, $(n, 2)$ sets have full Hausdorff dimension, Rev. Mat. Iberoamericana (to appear).

[Mi4] T. Mitsis, Topics in harmonic analysis, Reports University of Jyväskylä, Department of Mathematics and Statistics $\mathbf{8 8}$ (2003), 52pp.

[Mo] M. A. Monterie, Capacities of certain Cantor sets, Indag. Math. (N.S.) 8(2) (1997), 247-266.

[O] L. OLsEn, Geometric constructions in multifractal geometry, International Conference on Dimension and Dynamics (Miskolc, 1998), Period. Math. Hungar. 37(1-3) (1998), 81-99.

[O1] T. C. O'NeIL, A local version of the projection theorem, Proc. London Math. Soc. (3) 73(1) (1996), 68-104.

[O2] T. C. O'NeIL, The multifractal spectra of projected measures in Euclidean spaces, Chaos Solitons Fractals 11(6) (2000), 901-921.

[O3] T. C. O'NeIL, The Hausdorff dimension of the visible sets of connected compact sets, Preprint.

[P] H. РАJOT, "Analytic capacity, rectifiability, Menger curvature and the Cauchy integral", Lecture Notes in Mathematics 1799, Springer-Verlag, Berlin, 2002.

[PS] Y. PERES AND W. Schlag, Smoothness of projections, Bernoulli convolutions, and the dimension of exceptions, Duke Math. J. 102(2) (2000), 193-251. 
[PSS] Y. Peres, W. Schlag And B. Solomyak, Sixty years of Bernoulli convolutions, in: "Fractal geometry and stochastics, II" (Greifswald/Koserow, 1998), Progr. Probab. 46, Birkhäuser, Basel, 2000, pp. 39-65.

[PSS1] Y. Peres, K. Simon And B. Solomyak, Self-similar sets of zero Hausdorff measure and positive packing measure, Israel J. Math. 117 (2000), 353-379.

[PSS2] Y. Peres, K. Simon And B. Solomyak, Fractals with positive length and zero buffon needle probability, Amer. Math. Monthly 110(4) (2003), 314-325.

[PSo] Y. Peres And B. Solomyak, How likely is Buffon's needle to fall near a planar Cantor set?, Pacific J. Math. 204(2) (2002), 473-496.

[SY] T. D. SAuer And J. A. Yorke, Are the dimensions of a set and its image equal under typical smooth functions?, Ergodic Theory Dynam. Systems 17(4) (1997), 941-956.

[S1] P. SJlin, Estimates of spherical averages of Fourier transforms and dimensions of sets, Mathematika 40(2) (1993), 322-330.

[S2] P. SJLIN, Estimates of averages of Fourier transforms of measures with finite energy, Ann. Acad. Sci. Fenn. Math. 22(1) (1997), 227-236.

[S3] P. SJlin, Spherical harmonics and spherical averages of Fourier transforms, Rend. Sem. Mat. Univ. Padova 108 (2002), 41-51.

[SS1] P. SJLIN AND F. SoRIA, Some remarks on restriction of the Fourier transform for general measures, Publ. Mat. 43(2) (1999), 655-664.

[SS2] P. SJlin And F. Soria, Estimates of averages of Fourier transforms with respect to general measures, Preprint.

[S] B. Solomyak, On the random series $\sum \pm \lambda^{n}$ (an Erds problem), Ann. of Math. (2) 142(3) (1995), 611-625.

[T] M. TAlagrand, Sur la mesure de la projection d'un compact et certaines familles de cercles, Bull. Sci. Math. (2) 104(3) (1980), 225-231.

[Ta] T. TAO, From rotating needles to stability of waves: emerging connections between combinatorics, analysis, and PDE, Notices Amer. Math. Soc. 48(3) (2001), 294-303.

[To] X. Tolsa, Painlevé's problem and the semiadditivity of analytic capacity, Acta Math. 190(1) (2003), 105-149.

[W] B. White, A new proof of Federer's structure theorem for $k$-dimensional subsets of $\mathbb{R}^{N}$, J. Amer. Math. Soc. 11(3) (1998), 693-701. 
[W1] T. WolfF, Recent work connected with the Kakeya problem, in: "Prospects in mathematics" (Princeton, NJ, 1996), Amer. Math. Soc., Providence, RI, 1999, pp. 129-162.

[W2] T. WolfF, Decay of circular means of Fourier transforms of measures, Internat. Math. Res. Notices 10 (1999), 547-567.

[W3] T. WolfF, Addendum to "Decay of circular means of Fourier transforms of measures", J. Anal. Math. (to appear).

[Z] M. ZÄHLE, The average fractal dimension and projections of measures and sets in $\mathbf{R}^{n}$, Symposium in Honor of Benoit Mandelbrot (Curaao, 1995), Fractals 3(4) (1995), 747-754.

Department of Mathematics

University of Jyväskylä

P. O. Box 35

SF-40351 Jyväskylä

Finland

E-mail address: pmattila@maths.jyu.fi

Current address:

Department of Mathematics

P. O. Box 4 (Yliopistonkatu 5)

FIN-00014 University of Helsinki

Finland

E-mail address: pertti.mattila@helsinki.fi

Rebut el 25 d'abril de 2003. 